\title{
Some geometric invariants from resolutions of Hilbert modules
}

\author{
Ronald G. Douglas, Gadadhar Misra, and Cherian Varughese
}

\section{Introduction}

The model theory of Sz.-Nagy and Foias for contractions was reformulated in [15, chapter 3]. The existence of a unique minimal unitary dilation amounts to the existence of a Silov resolution for contractive Hilbert modules $\mathcal{M}$ over the disc algebra $\mathcal{A}(\mathbb{D})$ along with the fact that any two minimal Silov resolutions are isomorphic. The Silov modules appearing in the resolution may be classified using the von Neumann and Wold decomposition for isometries. This classification then allows one to identify a complete set of unitary invariants for the contractive module $\mathcal{M}$. This produces the characteristic function of Sz-Nagy and Foias which determines the contractive module up to unitary equivalence. Using localisation techniques and Beurling's Theorem [15, p. 115], it is possible to identify this characteristic function. If we wish to adapt this method for Hilbert modules over other function algebras, then first there is the question of existence of a resolution. Secondly, given a resolution, there is the question of identifying invariants for the module from the resolution. In the following, we will not address the first question but attempt to describe some possible invariants from a resolution. Having decided to ignore the question of existence of resolutions, we must identify situations where it is easy to write down a resolution. In this paper, we consider Hilbert spaces which consist of holomorphic functions on a bounded domain $\Omega \subseteq \mathbb{C}^{m}$ together with the natural action of the polynomials as multiplication operators. Thus we obtain a module $\mathcal{M}$ over the algebra of polynomials in $m$ variables. To continue our investigation, we must assume that the action is bounded which ensures that $\mathcal{M}$ is a module over the algebra $\mathcal{A}$ - the closure of the polynomials with respect to supremum norm on $\Omega$. If we consider the submodule $\mathcal{M}_{0}$ of all functions vanishing on a fixed subset $\mathcal{Z}$ of $\Omega$ then we obtain a short exact sequence

$$
0 \longleftarrow \mathcal{M}_{\mathrm{q}} \longleftarrow \mathcal{M} \stackrel{X}{\longleftarrow} \mathcal{M}_{0} \longleftarrow 0
$$

The research of the last two authors was partly carried out with financial support from the National Board for Higher Mathematics, India. 
of Hilbert modules, where $X$ is the inclusion map and the next arrow stands for the quotient map. There is another short exact sequence which occurs, namely,

$$
0 \longleftarrow \mathcal{Q} \longleftarrow \mathcal{M} \stackrel{X}{\longleftarrow} \mathcal{M} \longleftarrow 0,
$$

where $X$ is multiplication by some $\varphi$ in $\mathcal{A}$. Let $\mathcal{S}$ be the submodule determined by taking the closure of the range of this multiplication. Then $\mathcal{Q}$ is the quotient of $\mathcal{M}$ by the submodule $\mathcal{S}$. We see that the submodule $\mathcal{S}$ is contained in the submodule $\mathcal{M}_{0}$ of all functions vanishing on the zero set of $\varphi$. However, whether the two submodules $\mathcal{M}_{0}$ and $\mathcal{S}$ coincide, when $\mathcal{Z}$ is the zero set of $\varphi$, is probably a subtle question.

Let $\mathcal{M}_{0} \subseteq \mathcal{M}$ be a submodule and consider the resolution (1.1) of Hilbert modules over a function algebra $\mathcal{A}$. Over the past several years, we have attempted to obtain a canonical model and a complete set of unitary invariants for the quotient module $\mathcal{M}_{\mathrm{q}}$, at least in the case where $\mathcal{M}_{0}$ is assumed to be a submodule of functions vanishing on a hypersurface $\mathcal{Z} \subseteq \Omega$. To extract invariants from a resolution of the form (1.1), we assume that the module $\mathcal{M}$ is in the class $B_{1}\left(\Omega^{*}\right)$, where $\Omega^{*}=\left\{w \in \mathbb{C}^{m}: \bar{w} \in \Omega\right\}$. One may consult [15, p. 95] for more details about this class, some of which are also reproduced in the following section on Preliminaries. Then it is reasonable to expect that one should be able to use the existing theory to find the answer. Unfortunately, the submodule $\mathcal{M}_{0}$ does not necessarily belong to the same class making our quest somewhat tortuous. Here is an example.

EXAmple 1.1. Let $\mathcal{M}=H^{2}\left(\mathbb{D}^{2}\right)$ be the Hardy space on the bi-disc. This may be thought of as a Hilbert space of holomorphic functions defined on $\mathbb{D}^{2}$ determined by the reproducing kernel

$$
\hat{K}(z, w)=\left(1-z_{1} \bar{w}_{1}\right)^{-1}\left(1-z_{2} \bar{w}_{2}\right)^{-1}, z=\left(z_{1}, z_{2}\right), w=\left(w_{1}, w_{2}\right) \in \mathbb{D}^{2} .
$$

(We reserve the symbol $\hat{K}$ for the reproducing kernel of the Hardy space.)

Let $H_{0}^{2}\left(\mathbb{D}^{2}\right)=\left\{f \in H^{2}\left(\mathbb{D}^{2}\right): f(0,0)=0\right\}$ be the submodule $\mathcal{M}_{0}$ of functions vanishing at the origin. The reproducing kernel $\hat{K}_{0}(z, w)$ for $H_{0}^{2}\left(\mathbb{D}^{2}\right)$ is easily seen to be

$$
\hat{K}_{0}(z, w)=\left(z_{1} \bar{w}_{1}+z_{2} \bar{w}_{2}-z_{1} z_{2} \bar{w}_{1} \bar{w}_{2}\right) \hat{K}(z, w) .
$$

We verify that both the functions $z_{1}$ and $z_{2}$ lie in $\cap_{\ell=1}^{2}$ ker $P M_{z_{\ell}}^{*}$, where $P$ is the projection on to $H_{0}^{2}\left(\mathbb{D}^{2}\right)$. However, at all other points $w=\left(w_{1}, w_{2}\right) \in \mathbb{D}^{2}$, the joint kernel is spanned by $\hat{K}_{0}(\cdot, w)$. Consequently, we have $\operatorname{dim} \cap_{\ell=1}^{2} \operatorname{ker} P\left(M_{z_{\ell}}-w_{\ell}\right)^{*}=$ 1. The quotient module, in this case, is the one dimensional space spanned by the constant function 1 .

The assumption that $\mathcal{M}$ is in $B_{1}\left(\Omega^{*}\right)$ ensures the existence of an antiholomorphic, hermitian line bundle on the domain $\Omega$. The fiber of this bundle at $w \in \Omega$ is the joint kernel $\cap_{\ell=1}^{m} \operatorname{ker}\left(M_{\ell}-w_{\ell}\right)^{*}$, where $M_{\ell}$ is the operator induced by the module action of the generator $z_{\ell}$ in $\mathcal{A}$. It is then shown in [6] that the local equivalence of these bundles translates to unitary equivalence of elements in $B_{1}\left(\Omega^{*}\right)$. The curvature of this line bundle completely determines the unitary 
equivalence class. In the case of $H_{0}^{2}\left(\mathbb{D}^{2}\right)$, we can no longer associate a holomorphic hermitian vector bundle on all of $\mathbb{D}^{2}$. However, it is clear that the module is in $B_{1}\left(\mathbb{D}^{2} \backslash\{0\}\right)$ and therefore there exists a line bundle on $\mathbb{D}^{2} \backslash\{0\}$. This is the prototypical example of our discussion in the last section, where we indicate how to enlarge the domain of this bundle by "blowing up" the singularity at the origin. The invariants for the new line bundle defined on a larger domain then provide invariants for the submodule in question.

In [12], the submodule is taken to be the maximal set of functions vanishing on an analytic hypersurface $\mathcal{Z}$ in $\Omega$. Two descriptions are then provided for the quotient module. The first approach characterises it as a reproducing kernel Hilbert space. In the second approach, a hermitian, holomorphic line bundle on $\mathcal{Z}$ is associated with the quotient module. A complete unitary invariant for the quotient module is then the curvature of this line bundle. The submodule in [14] is taken to be the (maximal) set of functions which vanish to some given order $k$ on the hypersurface $\mathcal{Z}$. As in the previous case, two descriptions are provided for the quotient module. A matrix-valued kernel function must now be used and, in the vector bundle picture, we have a rank $k$ hermitian, holomorphic bundle. Some invariants for the quotient module (though not a complete set) are described. Some possible directions which might lead to other invariants are also discussed. In the section following the Preliminaries, we describe some of these results in detail along with concrete examples.

In [12] and [14], the approach chosen has been to use the resolution (1.1) of the quotient module and to construct invariants using the map $X$ (and metric information about some vector bundles determined by $\mathcal{M}$ and $\mathcal{M}_{0}$ ). It has, however, been shown in [13] that it may be fruitful to consider other resolutions of the quotient module. Let $\varphi$ be a defining function for $\mathcal{Z} \subseteq \Omega$. Assume that the closure of the range of multiplication by $\varphi$ on $\mathcal{M}$ coincides with the submodule $\mathcal{M}_{0}$ consisting of functions that vanish on the hypersurface $\mathcal{Z}$. Then it is shown that the following is a useful resolution:

$$
0 \longleftarrow \mathcal{M}_{\mathrm{q}} \longleftarrow \mathcal{M} \stackrel{M_{\varphi}}{\longleftarrow} \mathcal{M} \longleftarrow 0
$$

Here $M_{\varphi}$ is the operator of multiplication by $\varphi$. If $M_{\varphi}(w), w \in \Omega$ is the localisation of $M_{\varphi}$ (see subsection 2.2), it is shown that $\partial \bar{\partial} \log \left\|M_{\varphi}(w)\right\|^{2}$ represents the fundamental class of $\mathcal{Z}$. We point out here that the complex

$$
0 \longleftarrow \mathcal{M} \longleftarrow M_{\varphi} \mathcal{M} \longleftarrow 0
$$

is the simplest example of a Koszul complex and the sequence (1.3) is an extension of this Koszul complex by the quotient module.

More general Koszul complexes are useful tools in studying the case where $\mathcal{M}_{0}$ is taken to be the submodule of functions vanishing on an analytic submanifold $\mathcal{Z} \subseteq \Omega$ of codimension $r>1$. Some of the results obtained are briefly described below, the details are given in the later sections. 
Assume that $\mathcal{Z}$ is a complete intersection. $\mathcal{Z}$ is then the common zero set of $r$ defining functions $\varphi_{1}, \varphi_{2}, \cdots, \varphi_{r}$. It is possible to construct a useful resolution for the quotient module via a Koszul complex if we make the following further assumption on the submodule $\mathcal{M}_{0}$. Consider the range of the operator of multiplication by $\varphi_{j}, 1 \leq j \leq r$, defined on $\mathcal{M}$. It is clear that this range is contained in $\mathcal{M}_{0}$ for each $j$. We assume that $\mathcal{M}_{0}$ is the closure of the span of these ranges. Then it is possible to construct a resolution of the quotient module of length $r$ which allows us to identify the fundamental class of the submanifold $\mathcal{Z}$ using a generalisation of the Poincaré-Lelong equation due to Griffiths and King [17].

If $\varphi$ is the defining function for the hypersurface $\mathcal{Z}$, then the closure of the range of multiplication by $\varphi^{k}$ on $\mathcal{M}$ is a subspace of functions which vanish to order $k$ on $\mathcal{Z}$. Whether this is the largest subspace of functions with this property, as pointed out earlier, is a subtle question. In the absence of a definite answer to this question, we make the aparently restrictive assumption of the previous paragraph. We discuss some of these details in the third section.

An alternative resolution of the quotient module is by means of a grid and it is possible to determine the fundamental class of $\mathcal{Z}$ as a product of $(1,1)$ currents (cf. [8]) obtained from this resolution. We have described the simplest case of $r=2$ (see (4.8)) in some detail in this paper. Although working out the details of the "grid construction" in the general case is not entirely trivial, the results are similar.

\section{Preliminaries}

We begin by describing the standing assumptions that we make all through this paper. By a hypersurface $\mathcal{Z} \subseteq \Omega$ we mean a complex submanifold of dimension $m-1$. It follows that, given $z^{(0)} \in \mathcal{Z}$, there is a neighborhood $U \subseteq \Omega$ and a holomorphic map $\varphi: U \rightarrow \mathbb{C}$ such that $\left(\partial \varphi / \partial z_{j}\right)\left(z^{(0)}\right) \neq 0$ for some $j$, with $1 \leq j \leq m$ and

$$
U \cap \mathcal{Z}=\{z \in U: \varphi(z)=0\} .
$$

In this case, we say that $\varphi$ is a defining function for the hypersurface $\mathcal{Z}$. Whenever we discuss a zero variety, we will assume that it is the common zero set of holomorphic functions $\varphi_{1}, \ldots, \varphi_{n}$ defined on $\Omega$. It must be pointed out that our interest lies in equivalence classes of modules consisting of holomorphic functions defined on $\Omega$. The restriction of such modules to an open subset $U$ of $\Omega$ yield equivalent modules as pointed out in [14, p. 370]. Consequently, we can (by going to a smaller open set if necessary) ensure that the zero variety has the description given above. The only assumption we make is that if the zero variety is the intersection of a number of hypersurfaces, then it must be a complete intersection. The assumption of complete intersection allows us to get around a number of technical difficulties - none very serious. We hope to study the general case in the near future.

Let $\Omega \subseteq \mathbb{C}^{m}$ be a bounded, simply connected domain in $\mathbb{C}^{m}$. Let $\mathcal{A}(\Omega)$ denote the closure with respect to the supremum norm on $\Omega$ of functions holomorphic in a neighborhood of $\bar{\Omega}$, the closure of the domain $\Omega \subseteq \mathbb{C}^{m}$. Then $\mathcal{A}(\Omega)$ is a function 
algebra and consists of continuous functions on $\bar{\Omega}$ which are holomorphic on $\Omega$. We assume that $\Omega$ is polynomially convex which then ensures that $\mathcal{A}(\Omega)$ is the closure of polynomials with respect to the supremum norm on $\Omega$.

Let $\mathcal{M}$ be a Hilbert module over $\mathcal{A}(\Omega)$. We assume that the point evaluation $f \mapsto f(w), f \in \mathcal{M}$ is bounded for each $w \in \Omega$. Consequently, $\mathcal{M}$ admits a reproducing kernel $K: \Omega \times \Omega \rightarrow \mathbb{C}$. We assume, in addition, that $\mathcal{M}$ consists of holomorphic functions on $\Omega$ and contains all the polynomials, and lies in the Cowen-Douglas class $B_{1}\left(\Omega^{*}\right)$, that is, $\left(M_{1}^{*}, \ldots, M_{m}^{*}\right)$ is in $B_{1}\left(\Omega^{*}\right)$. Although it may not be absolutely necessary for what follows, we also assume that $\mathcal{M}$ is of rank 1 .

2.1. Functional Hilbert spaces and $B_{k}(\Omega)$

Let $E$ be a $k$-dimensional Hilbert space and $\mathcal{L}(E)$ denote the vector space of all linear transformations on $E$. A function $K: \Omega \times \Omega \rightarrow \mathcal{L}(E)$, satisfying

$$
\sum_{i, j=1}^{n}\left\langle K\left(w_{i}, w_{j}\right) \zeta_{j}, \zeta_{i}\right\rangle_{E} \geq 0, w_{1}, \ldots, w_{n} \in \Omega, \zeta_{1}, \ldots, \zeta_{n} \in E, n \geq 1
$$

is said to be a non negative definite (nnd) kernel on $\Omega$. Given such an nnd kernel $K$ on $\Omega$, it is easy to construct a Hilbert space $\mathcal{H}$ of functions on $\Omega$ taking values in $\mathrm{E}$ with the property

$$
\langle f(w), \zeta\rangle_{E}=\langle f, K(\cdot, w) \zeta\rangle_{\mathcal{H}}, w \in \Omega, \zeta \in E, f \in \mathcal{H}
$$

The Hilbert space $\mathcal{H}$ is simply the completion of the linear span of all vectors of the form $K(\cdot, w) \zeta, w \in \Omega, \zeta \in E$, with inner product defined by (2.2).

Conversely, let $\mathcal{H}$ be any Hilbert space of functions on $\Omega$ taking values in $E$. Let $e_{w}: \mathcal{H} \rightarrow E$ be the evaluation functional defined by $e_{w}(f)=f(w), w \in \Omega$, $f \in \mathcal{H}$. If $e_{w}$ is bounded for each $w \in \Omega$ then it is easy to verify that the Hilbert space $\mathcal{H}$ possesses a reproducing kernel $K(z, w)=e_{z} e_{w}^{*}$, that is, $K(\cdot, w) \zeta \in \mathcal{H}$ for each $w \in \Omega$ and $K$ has the reproducing property (2.2). Finally, the reproducing property (2.2) determines the reproducing kernel $K$ uniquely.

REMARK 2.1. If we assume that $\mathcal{H}$ is a Hilbert space consisting of holomorphic functions taking values in $E$, then it is not hard to see that the map $w \mapsto e_{w}$ is weak-* holomorphic. Hence it is also strongly holomorphic as a map into the dual of $\mathcal{H}$. But since $\left(\partial_{i} e_{w}\right)(h)=\left(\partial_{i} h\right)(w)$, for $h \in \mathcal{H}$ and $w \in \Omega$, it follows that evaluations of partial derivatives induce bounded linear functionals on $\mathcal{H}$. Therefore, we see that $\bar{\partial}_{i} K(\cdot, w)$ is in $\mathcal{H}$, where $\bar{\partial}_{i}=\frac{\partial}{\partial \bar{w}_{i}}$.

Let $T=\left(T_{1}, \ldots, T_{m}\right)$ be an $m$-tuple of commuting bounded linear operators on a separable complex Hilbert space $\mathcal{H}$. Define the operator $D_{T}: \mathcal{H} \rightarrow$ $\mathcal{H} \oplus \cdots \oplus \mathcal{H}$ by $D_{T}(x)=\left(T_{1} x, \ldots, T_{m} x\right), x \in \mathcal{H}$. For $w=\left(w_{1}, \ldots, w_{m}\right) \in \Omega$, let $T-w$ denote the operator tuple $\left(T_{1}-w_{1}, \ldots, T_{m}-w_{m}\right)$. Let $k$ be a positive integer.

Definition 2.1. The $m$-tuple $T$ is said to be in the Cowen - Douglas class $B_{k}(\Omega)$ if 
1. $\operatorname{ran} D_{T-w}$ is closed for all $w \in \Omega$

2. $\operatorname{span}\left\{\operatorname{ker} D_{T-w}: w \in \Omega\right\}$ is dense in $\mathcal{H}$

3. $\operatorname{dim} \operatorname{ker} D_{T-w}=k$ for all $w \in \Omega$.

This class was introduced in [7]. The case of a single operator was investigated earlier in the paper [6], where it is pointed out that an operator $T$ in $B_{1}(\Omega)$ is unitarily equivalent to the adjoint of the multiplication operator $M$ on a reproducing kernel Hilbert space. Here $(M f)(z)=z f(z), f \in \mathcal{H}$. It is not very hard to see that, more generally, an $m$-tuple $T$ in $B_{k}(\Omega)$ is unitarily equivalent to the adjoint of the $m$-tuple of multiplication operators $M=\left(M_{1}, \ldots, M_{m}\right)$ on a reproducing kernel Hilbert space [10, Remark 2.6 a) and b)]. Conversely, Curto and Salinas [10] show that if certain conditions are imposed on the reproducing kernel, then the corresponding adjoint of the $m$-tuple of multiplication operators belongs to the class $B_{k}(\Omega)$.

Let $T$ be an $m$-tuple of operators in $B_{k}(\Omega)$ on $\mathcal{H}$. Pick $k$ linearly independent vectors $\gamma_{1}(w), \ldots, \gamma_{k}(w)$ in $\operatorname{ker} D_{T-w}, w \in \Omega$. Define a map $\Gamma: \Omega \rightarrow \mathcal{L}\left(\mathbb{C}^{k}, \mathcal{H}\right)$ by $\Gamma(w) \zeta=\sum_{i=0}^{k} \zeta_{i} \gamma_{i}(w)$, where $\zeta=\left(\zeta_{1}, \ldots, \zeta_{k}\right) \in \mathbb{C}^{k}$. It is shown in [6, Proposition 1.11] and [10, Theorem 2.2] that it is possible to choose $\gamma_{1}(w), \ldots, \gamma_{k}(w), w$ in some domain $\Omega_{0} \subseteq \Omega$, such that $\Gamma$ is holomorphic on $\Omega_{0}$. Let $\mathcal{A}\left(\Omega, \mathbb{C}^{k}\right)$ denote the linear space of all $\mathbb{C}^{k}$ - valued holomorphic functions on $\Omega$. Define $U_{\Gamma}: \mathcal{H} \rightarrow \mathcal{A}\left(\Omega_{0}^{*}, \mathbb{C}^{k}\right)$ by

$$
\left(U_{\Gamma} x\right)(w)=\Gamma(w)^{*} x, x \in \mathcal{H}, w \in \Omega_{0} .
$$

Define a sesqui-linear form on $\mathcal{H}_{\Gamma}=\operatorname{ran} U_{\Gamma}$ by $<U_{\Gamma} f, U_{\Gamma} g>_{\Gamma}=<f, g>, f, g \in \mathcal{H}$. The map $U_{\Gamma}$ is linear and injective. Hence $\mathcal{H}_{\Gamma}$ is a Hilbert space of $\mathbb{C}^{k}$-valued holomorphic functions on $\Omega_{0}^{*}$ with inner product $<\cdot, \cdot>_{\Gamma}$ and $U_{\Gamma}$ is unitary. Then it is easy to verify the following (cf. [10, Remarks 2.6]).

a): $K(z, w)=\Gamma(\bar{z})^{*} \Gamma(\bar{w}), z, w \in \Omega_{0}^{*}$ is the reproducing kernel for the Hilbert space $\mathcal{H}_{\Gamma}$.

b): $M_{i}^{*} U_{\Gamma}=U_{\Gamma} T_{i}$, where $\left(M_{i} f\right)(z)=z_{i} f(z), z \in \Omega$.

c): There exists $w_{0} \in \Omega_{0}^{*}$ such that $K\left(z, w_{0}\right)=I$ for all $z \in \Omega_{0}^{*}$.

An nnd kernel satisfying the condition c) above is said to be normalised at $w_{0}$.

Conversely, it is possible to impose conditions on a kernel function $K$ : $\Omega \times \Omega \rightarrow \mathcal{L}\left(\mathbb{C}^{k}\right)$ ensuring the boundedness of each of the multiplication operators $M_{1}, \ldots, M_{m}$ on the associated reproducing kernel Hilbert space. One may impose additional conditions on $K$ to ensure that $M$ is in $B_{k}\left(\Omega^{*}\right)$.

To an $m$-tuple $T$ in $B_{k}(\Omega)$, on the one hand, one may associate a holomorphic, hermitian vector bundle on $\Omega$ (cf. [6]), which is obtained as the pullback of the universal bundle on the Grassmanian $G r(\mathcal{H}, k)$ of the Hilbert space $\mathcal{H}$ under the map $\gamma: \Omega \rightarrow G r(\mathcal{M}, k)$, where $\gamma(w)=\operatorname{ker} D_{T-w}$. On the other hand, one may associate a normalised reproducing kernel (cf. [10]) on a suitable subdomain of $\Omega^{*}=\left\{w \in \mathbb{C}^{m}: \bar{w} \in \Omega\right\}$. It is possible to answer a number of questions regarding the $m$-tuple of operators $T$ using either the vector bundle or the reproducing kernel. 


\subsection{Localisation}

Let $\mathcal{M}$ and $\mathcal{N}$ be any two Hilbert modules over the algebra $\mathcal{A}$. Notice that there are two possible module actions on $\mathcal{M} \otimes \mathcal{N}$, i.e., the left action: $L \otimes I:(f, h \otimes k) \mapsto$ $f \cdot h \otimes k$ and the right action: $I \otimes R:(f, h \otimes k) \mapsto h \otimes f \cdot k$. The module tensor product $\mathcal{M} \otimes_{\mathcal{A}} \mathcal{N}$ is defined to be the module obtained by identifying these two actions. Specifically, let $\mathcal{E}$ be the closed subspace of $\mathcal{M} \otimes \mathcal{N}$ generated by vectors of the form

$$
\{f \cdot h \otimes k-h \otimes f \cdot k: h \in \mathcal{M}, k \in \mathcal{N} \text { and } f \in \mathcal{A}\} .
$$

Then $\mathcal{E}$ is a submodule of $\mathcal{M} \otimes \mathcal{N}$ with respect to both the left and the right actions. The module tensor product $\mathcal{M} \otimes_{\mathcal{A}} \mathcal{N}$ is defined to be $(\mathcal{M} \otimes \mathcal{N}) \ominus \mathcal{E}$ together with the compression of either the left or the right actions, which coincide on this space. For fixed $w \in \Omega, \mathbb{C}$ is a module over $\mathcal{A}$ with the module action

$$
(f, v) \mapsto f(w) v, f \in \mathcal{A}, v \in \mathbb{C} .
$$

Let $\mathbb{C}_{w}$ denote the one dimensional module $\mathbb{C}$ with this action. We will largely confine ourselves to the module tensor product $\mathcal{M} \otimes_{\mathcal{A}} \mathbb{C}_{w}$, which we denote by $\mathcal{M}(w)$.

REMARK 2.2. Let $\mathcal{M}$ be a module over the algebra $\mathcal{A}(\Omega)$. Then the subspace $\mathcal{E}$ of $\mathcal{M} \otimes \mathbb{C}_{w}$ is easily seen to be

$$
\mathcal{E}=\operatorname{span}\{((f-f(w)) \cdot h) \otimes 1: f \in \mathcal{A}(\Omega), h \in \mathcal{M}\} .
$$

It is therefore clear that the localisation $\mathcal{M}(w)$ at $w$, which is $\mathcal{E}^{\perp}$ is the common eigenspace of the "adjoint action" at $w$, that is,

$$
\mathcal{M}(w)=\cap_{f \in \mathcal{A}} \operatorname{ker}\left(M_{f}-f(w)\right)^{*} .
$$

The action of $f \in \mathcal{A}$ on $\mathcal{M}(w)$ is scalar and is determined by $f(w)$.

If we localise the submodule $H_{0}^{2}\left(\mathbb{D}^{2}\right)$ of Example 1.1, using the module $\mathbb{C}_{w}$, where $w=\left(w_{1}, w_{2}\right) \in \mathbb{D}^{2}$, then we find that

$$
H_{0}^{2}\left(\mathbb{D}^{2}\right) \otimes_{\mathcal{A}\left(\mathbb{D}^{2}\right)} \mathbb{C}_{w}= \begin{cases}\mathbb{C}_{0} \oplus \mathbb{C}_{0} & w=(0,0) \\ \mathbb{C}_{w} & w \neq(0,0) .\end{cases}
$$

A less trivial example is obtained as follows.

EXAMPLE 2.1. Let $M$ be the multiplication operator on the Hardy space $H^{2}(\mathbb{D})$ on the disc. Consider the action of the bi-disc algebra $\mathcal{A}\left(\mathbb{D}^{2}\right)$ on the Hardy space $H^{2}(\mathbb{D})$ induced by the pair $\left(M^{2}, M^{3}\right)$. Now, the Hardy space $H^{2}(\mathbb{D})$ is 2 - generated, as a Hilbert module over $\mathcal{A}\left(\mathbb{D}^{2}\right)$, by 1 and $z$. If we localise $H^{2}(\mathbb{D})$ using the module $\mathbb{C}_{w}$, where $w=\left(w_{1}, w_{2}\right) \in \mathbb{D}^{2}$, then we find that

$$
H^{2}(\mathbb{D}) \otimes_{\mathcal{A}\left(\mathbb{D}^{2}\right)} \mathbb{C}_{w}= \begin{cases}\mathbb{C}_{0} \oplus \mathbb{C}_{0} & w=(0,0) \\ \mathbb{C}_{w} & w=\left(\alpha^{2}, \alpha^{3}\right), \alpha \neq 0, \alpha \in \mathbb{D} \\ (0) & w \neq\left(\alpha^{2}, \alpha^{3}\right), \alpha \in \mathbb{D} .\end{cases}
$$


If we remove the function $z$ by considering the subspace $H_{1}^{2}(\mathbb{D})$ of $H^{2}(\mathbb{D})$ spanned by $\left\{1, z^{2}, \ldots\right\}$, then $H_{1}^{2}(\mathbb{D})$ is also a contractive Hilbert module over $\mathcal{A}\left(\mathbb{D}^{2}\right)$, and now

$$
H_{1}^{2}(\mathbb{D}) \otimes_{\mathcal{A}\left(\mathbb{D}^{2}\right)} \mathbb{C}_{w}= \begin{cases}\mathbb{C}_{w} & w=\left(\alpha^{2}, \alpha^{3}\right), \alpha \in \mathbb{D} \\ (0) & w \neq\left(\alpha^{2}, \alpha^{3}\right), \alpha \in \mathbb{D} .\end{cases}
$$

If $X$ is a map from a module $\mathcal{M}$ to a module $\mathcal{N}$, we denote by $X(w)$ the restriction of $X \otimes I$ (defined on $\mathcal{M} \otimes \mathbb{C}_{w}$ ) to $\mathcal{M}(w)$ followed by the projection onto $\mathcal{N}(w)$.

A Hilbert module $\mathcal{M}$ over an algebra $\mathcal{A}(\Omega)$ is said to be of rank $k$ if there exists $\Gamma \subseteq \mathcal{M}$ of cardinality $k$ such that the linear span of $\{f \cdot h: f \in \mathcal{A}(\Omega)$ and $h \in \Gamma\}$ is dense in $\mathcal{M}$, and no subset of $\mathcal{M}$ of smaller cardinality has this property.

If $\mathcal{M}$ is a module of rank 1 , then we find (cf. [15, Lemma 5.1]) that $\operatorname{dim} \mathcal{M}(w)$, $w \in \Omega$, is at most 1 .

Recall that $\mathcal{M}$ is said to be locally free if the sheaf determined by the localisation $\mathcal{M}(w)$ defines a finite dimensional holomorphic, hermitian vector bundle over $\Omega$ and the linear span of the fibers is dense in $\mathcal{M}$.

We point out that locally free modules over $\mathcal{A}(\Omega)$ of finite rank, say $k$, resemble modules in the class $B_{\ell}(\Omega)$, for some $\ell \leq k$.

\subsection{Factorisation of the reproducing kernel for the submodule $\mathcal{M}_{0}$}

We recall from [14, Lemma 2.6] that if $\mathcal{M}_{0}$ is the submodule of functions vanishing on an irreducible hypersurface $\mathcal{Z} \subseteq \Omega$, then the reproducing kernel $K_{0}$ of $\mathcal{M}_{0}$ admits the following factorisation:

$$
K_{0}(z, w)=\varphi(z) \chi(z, w) \overline{\varphi(w)}, z, w \in \Omega,
$$

where $\varphi$ is a defining function for $\mathcal{Z}$ and $\chi: \Omega \times \Omega \rightarrow \mathbb{C}$ is some function holomorphic in the first variable and anti-holomorphic in the second one. Moreover, $\chi(w, w) \neq 0$ for any $w \in \Omega$. Since $\varphi$ is a defining function, we may assume, without loss of generality, that $\partial_{1} \varphi(w) \neq 0$, for $w \in \Omega$. Clearly,

$$
\left(\bar{\partial}_{1} K_{0}\right)(\cdot, w)=\varphi(\cdot) \chi(\cdot, w) \overline{\left(\partial_{1} \varphi\right)(w)} \text { for } w \in \mathcal{Z} .
$$

Notice that $\bar{\partial}_{1} K_{0}(\cdot, w)$, in view of Remark 2.1, is in $\mathcal{M}_{0}$. Now, it follows from the reproducing property of $K_{0}$ that

$$
M_{f}^{*}\left(\bar{\partial}_{1} K_{0}\right)(\cdot, w)=\overline{\left(\partial_{1} f\right)(w)} K_{0}(\cdot, w)+\overline{f(w)}\left(\bar{\partial}_{1} K_{0}\right)(\cdot, w), f \in \mathcal{A} .
$$

The fact that $\left(\bar{\partial}_{1} K_{0}\right)(\cdot, w)$ is a non zero vector for $w \in \mathcal{Z}$ in $\mathcal{M}_{0}$ follows from equation (2.5). Therefore, we conclude that $\left(\bar{\partial}_{1} K_{0}\right)(\cdot, w)$ is an eigenvector for $M_{f}^{*}$ as long as $w \in \mathcal{Z}$. At this juncture, if we assume that the submodule $\mathcal{M}_{0}$ coincides with the range closure of the operator $M_{\varphi}$, then it will follow that $\mathcal{M}_{0}$ is of rank 1 (we are making the standing assumption that $\mathcal{M}$ is of rank 1 ). We then see that $\mathcal{M}_{0}$ is a locally free module of rank 1 .

Let $\Gamma: \Omega \rightarrow \mathcal{M}_{0}$ be the map defined by $\Gamma(w)=\varphi(\cdot) \chi(\cdot, w)$, then using the reproducing property of $K_{0}$ and (2.5), we find that

$$
<\Gamma(w), \Gamma\left(w^{\prime}\right)>=\chi\left(w^{\prime}, w\right) .
$$


Therefore, following the discussion in section 2.1 , we see that the module $\mathcal{M}_{0}$ is isomorphic to a Hilbert module consisting of holomorphic functions on $\Omega$ whose reproducing kernel is $\chi$. We have therefore proved:

Proposition 2.1. Let $\mathcal{M}$ be a Hilbert module of rank 1 and $\mathcal{M}_{0}$ be a submodule of functions as above (among other things, $\mathcal{M}_{0}$ coincides with the range closure of the operator $\left.M_{\varphi}\right)$. Then $\mathcal{M}_{0}$ is locally free and is of rank 1 . Moreover, the submodule $\mathcal{M}_{0}$ is isomorphic to a module of holomorphic functions on $\Omega$ whose reproducing kernel is $\chi$.

REMARK 2.3. We point out that the assumption of irreducibility of the hypersurface is superfluous for the kernel of the submodule $\mathcal{M}_{0}$ to admit a factorisation of the form (2.4) as long as the submodule is the closure in $\mathcal{M}$ of a principal ideal $\mathcal{I}$. We may then take $\varphi$ to be the generator of this ideal and obtain a factorisation formula exactly as in (2.4) under the additional hypothesis that $\mathcal{M}_{0}$ equals the space of all functions vanishing on the common zero set of the ideal $\mathcal{I}$. The only difference is that, in this case, the generator $\varphi$ of the ideal $\mathcal{I}$ is not necessarily irreducible and admits a factorisation $\varphi=\varphi_{1} \cdots \varphi_{\ell}$ into irreducible factors.

We describe an obvious example. Let $H_{12}^{2}\left(\mathbb{D}^{2}\right) \subseteq H^{2}\left(\mathbb{D}^{2}\right)$ be the submodule of functions vanishing on the set $\left\{\left(z_{1}, z_{2}\right) \in \mathbb{D}^{2}: z_{1}=0\right.$ or $\left.z_{2}=0\right\}$. Of course, this submodule is also the closure of the ideal $\mathcal{I}_{12} \subseteq H^{2}\left(\mathbb{D}^{2}\right)$ generated by $z_{1} z_{2}$. In this case, the reproducing kernel for the submodule is easily seen to be

$$
K_{0}(z, w)=z_{1} \bar{w}_{1} z_{2} \bar{w}_{2} \hat{K}(z, w), z=\left(z_{1}, z_{2}\right), w=\left(w_{1}, w_{2}\right) \in \mathbb{D}^{2}
$$

This is a locally free module of rank 1.

We now consider the case where the submodule $\mathcal{M}_{0}$ consists of functions vanishing on the intersection of two hypersurfaces $\mathcal{Z}_{1}$ and $\mathcal{Z}_{2}$. Let $\varphi_{1}$ and $\varphi_{2}$ be the defining functions (on a common open set) for these two hypersurfaces. Let $\mathcal{M}_{1}$ and $\mathcal{M}_{2}$ be the two submodules of functions vanishing on these hypersurfaces and $\mathcal{M}_{12}$ be the submodule of functions vanishing on the set $\mathcal{Z}_{1} \cup \mathcal{Z}_{2}$ which is $\mathcal{M}_{1} \cap \mathcal{M}_{2}$. It is clear that

$$
\mathcal{M}_{0}=\left(\mathcal{M}_{1} \ominus \mathcal{M}_{12}\right) \oplus\left(\mathcal{M}_{2} \ominus \mathcal{M}_{12}\right) \oplus \mathcal{M}_{12}
$$

We have already shown (recall Proposition 2.1) that the reproducing kernels $K_{\ell}(z, w)$, corresponding to the submodules $\mathcal{M}_{\ell}, \ell=1,2$, admit factorisations. Notice that $\mathcal{M}_{12}$ consists of functions which vanish on the common zero set of the function $\varphi_{1} \varphi_{2}$. The preceding remark ensures that a similar factorisation exists for $\mathcal{M}_{12}$ as well. Therefore, we obtain the following formula for the reproducing 
kernel $K_{0}$ corresponding to the submodule $\mathcal{M}_{0}$ :

$$
\begin{aligned}
K_{0}(z, w)= & \left(\varphi_{1}(z) \chi_{1}(z, w) \overline{\varphi_{1}(w)}-\varphi_{1}(z) \varphi_{2}(z) \chi_{12}(z, w) \overline{\varphi_{1}(w) \varphi_{2}(w)}\right) \\
& +\left(\varphi_{2}(z) \chi_{2}(z, w) \overline{\varphi_{2}(w)}-\varphi_{1}(z) \varphi_{2}(z) \chi_{12}(z, w) \overline{\varphi_{1}(w) \varphi_{2}(w)}\right) \\
& +\varphi_{1}(z) \varphi_{2}(z) \chi_{12}(z, w) \overline{\varphi_{1}(w) \varphi_{2}(w)} \\
= & \varphi_{1}(z) \chi_{1}(z, w) \overline{\varphi_{1}(w)}+\varphi_{2}(z) \chi_{2}(z, w) \overline{\varphi_{2}(w)} \\
& -\varphi_{1}(z) \varphi_{2}(z) \chi_{12}(z, w) \overline{\varphi_{1}(w) \varphi_{2}(w)}
\end{aligned}
$$

where $\chi_{\ell}(w, w) \neq 0$ for $w \in \mathcal{Z}_{\ell}, \ell=1,2$ and $\chi_{12}(w, w) \neq 0$ for $w \in \mathcal{Z}_{1} \cap \mathcal{Z}_{2}$. The formula for the reproducing kernel $\hat{K}_{0}$ which was given in Example 1.1 is an instance of the general formula given above with $\varphi_{\ell}(z)=z_{\ell}, \ell=1,2$.

REMARK 2.4. In the case of a submodule $\mathcal{M}_{0}$ of functions vanishing on a hypersurface, although the reproducing kernel vanishes on this hypersurface, Proposition 2.1 shows how to construct an isomorphic copy of this module with a non-vanishing reproducing kernel. This amounts to dividing by $\varphi$. The resulting kernel $\chi$ may then be used to construct a bundle, via the map $w \mapsto \chi(\cdot, w)$, on all of $\Omega$. However, in the case where the submodule consists of functions vanishing on the intersection of two hypersurfaces, as above, if we attempt to divide either by $\varphi_{1}$ or $\varphi_{2}$, then the limit as we approach the zero set may not exist. However, we can still do this as long as we are willing to enlarge the domain. The details of this "blow up" construction are discussed in the last section.

\section{The case of a principal ideal}

Assuming that the zero variety $\mathcal{Z}$ is a hypersurface, we have been able to obtain complete unitary invariants for the quotient modules only under the further assumption that $\mathcal{M}_{0}$ consists of the maximal set of functions in $\mathcal{M}$ which vanish on $\mathcal{Z}$. Even in the case when $\mathcal{Z}$ is not assumed to be a hypersurface, the quotient module is, surprisingly, the restriction of the module $\mathcal{M}$ to $\mathcal{Z}$, as long as $\mathcal{M}_{0}$ continues to be the maximal set of functions in $\mathcal{M}$ which vanish on $\mathcal{Z}$. If $\mathcal{M}$ is assumed to be in $B_{1}\left(\Omega^{*}\right)$, then the quotient module can once again be described by a line bundle on $\mathcal{Z}$. The restriction of the curvature then provides a complete unitary invariant for the quotient module. Therefore, one may argue that there is no need to find any other invariant for these quotients modules. Our aim in obtaining such invariants in the case which we understand reasonably well is the hope that some analogues may be found in the case where we drop the assumption that $\mathcal{M}_{0}$ consists of the maximal set of functions in $\mathcal{M}$ which vanish on $\mathcal{Z}$. For instance, we have found an analogue of the alternating sum (4.1) in [14, Theorem 4.4]. However, we have not succeeded in evaluating this current. There is almost certainly a possibility of applying the methods developed by Harvey and Lawson [19] to evaluate this current which we propose to undertake during the course of our work. 
We describe one instance which we have studied in some detail. Let $\mathcal{M}_{0}$ consist of functions vanishing to order $k$ on a hyper-surface $\mathcal{Z}$ in $\Omega$. Following our assumption above, $\mathcal{M}_{0}$ is the closure of a principal ideal in $\mathcal{M}$.

As mentioned earlier, in the case where $\mathcal{M}_{0}$ consists of functions in $\mathcal{M}$ that vanish to order $k$, a description of the quotient module is presented in [14]. A brief description of the model is presented here.

Starting with the module $\mathcal{M}$, we construct a unitarily equivalent module, denoted $J \mathcal{M}$, using the map $J$ defined as follows:

$$
J: h \mapsto \sum_{j=0}^{k-1} \partial_{1}^{j} h \otimes \epsilon_{j+1} .
$$

Here $\left\{\epsilon_{j}\right\}_{j=1}^{k}$ is the standard basis in $\mathbb{C}^{k}$ and $\partial_{1}$ denotes the derivative with respect to $z_{1}$ if the zero set is characterised, in local coordinates, by the equation $z_{1}=0$. Hence $J \mathcal{M}$ is a vector-valued Hilbert space and the norm on it is determined by the unitarity of the map $J$.

The module action on $J \mathcal{M}$ is given in matrix form by $f \mapsto \mathcal{J} f$, for $f \in \mathcal{A}(\Omega)$, where

$$
\mathcal{J}=\left(\begin{array}{ccccc}
1 & & & & \\
& \ddots & & 0 & \\
\vdots & \left(\begin{array}{l}
\ell \\
j
\end{array}\right) \partial_{1}^{\ell-j} & 1 & & \\
& & & \ddots & \\
\partial_{1}^{k-1} & & \ldots & & 1
\end{array}\right)
$$

with $0 \leq \ell, j \leq k-1$.

We now construct a module over the algebra $\mathcal{A}(\Omega)$ as follows. The Hilbert space denoted $(J \mathcal{M})_{\text {res }}$ is obtained by restricting the elements of $J \mathcal{M}$ to the zero set, with norm given by

$$
\left\|\mathbf{h}_{0}\right\|=\inf \left\{\|\mathbf{h}\|:\left.\mathbf{h}\right|_{\mathcal{Z}}=\mathbf{h}_{0} \text { for } \mathbf{h} \in J \mathcal{M}\right\} .
$$

The module action is taken to be

$$
\left.\left.\left(f,\left.\mathbf{h}\right|_{\mathcal{Z}}\right) \mapsto \mathcal{J} f\right|_{\mathcal{Z}} \cdot \mathbf{h}\right|_{\mathcal{Z}}
$$

for $f \in \mathcal{A}(\Omega)$.

The reproducing kernel for $(J \mathcal{M})_{\text {res }}$ is obtained by restricting the reproducing kernel of $J \mathcal{M}$ in both arguments. We denote the resulting reproducing kernel by $(J K)_{\text {res }}$.

We reproduce below the characterisation of the quotient module $[14$, cf. Theorem 3.3].

THEOREM 3.1. Let $\mathcal{M}$ be a Hilbert module over the algebra $\mathcal{A}(\Omega)$ and let $\mathcal{M}_{0}$ be the submodule of functions $h$ such that $\partial_{1}^{j} h$ vanishes on $\mathcal{Z}$ for $0 \leq j \leq k-1$. The quotient module is then equivalent to $(J \mathcal{M})_{\text {res }}$. 
The above description of the quotient module is sufficiently general and encompasses some earlier descriptions of quotient modules that were worked out in specific examples. We illustrate this by means of some examples.

EXAmple 3.1. Let $\mathcal{M}$ be the space $H^{2}\left(\mathbb{D}^{2}\right)$ as a module over the bi-disc algebra $\mathcal{A}\left(\mathbb{D}^{2}\right)$ and let $\mathcal{M}_{0}$ be the maximal subset of functions that vanish along the diagonal $z_{1}=z_{2}$, where $\left(z_{1}, z_{2}\right)$ are coordinates on $\mathbb{D}^{2}$. The quotient module is spanned by the (normalised) collection $\left\{e_{k}\right\}_{k=0}^{\infty}$ of homogeneous polynomials defined by

$$
e_{k}\left(z_{1}, z_{2}\right)=\frac{1}{\sqrt{k+1}} \sum_{j=0}^{k} z_{1}^{k-j} z_{2}^{j}
$$

The module actions of $z_{1}$ and $z_{2}$ are the same and are described by the map

$$
e_{k} \mapsto \sqrt{\frac{k+1}{k+2}} e_{k+1}
$$

The general picture described above by restricting functions to the zero set yields the Bergman space on the disc. The coordinates $z_{1}$ and $z_{2}$ are now identified and the corresponding module action is the multiplication operator on the Bergman space. The unitary map that intertwines the two pictures is the one that maps $e_{k}$ to $\frac{z^{k}}{\sqrt{k+1}}$ in the Bergman space.

We point out that some generalisations of the above example have been studied by D. N. Clark in [5]. A more general family of zero sets is studied there, of which the diagonal in $\mathbb{D}^{2}$ is a special case.

EXAMPLE 3.2 (Continuation of Example 2.1). The model of the quotient module as functions obtained by restricting to the zero set is a useful one. The following example further emphasises this fact. Let $\mathcal{M}$ be the module $H^{2}\left(\mathbb{D}^{2}\right)$ over the algebra $\mathcal{A}\left(\mathbb{D}^{2}\right)$. Let $\mathcal{M}_{0}$ be the submodule consisting of functions which vanish on the zero set determined by the function $z_{1}^{3}-z_{2}^{2}$. Thus we obtain a resolution

$$
0 \longleftarrow \mathcal{M}_{\mathrm{q}} \longleftarrow \mathcal{M} \stackrel{X}{\longleftarrow} \mathcal{M}_{0} \longleftarrow 0 .
$$

The zero set is one (complex) dimensional and a parametrised description of it as a subset of $\mathbb{D}^{2}$ is the collection of points in $\mathbb{D}^{2}$ of the form $\left(\alpha^{2}, \alpha^{3}\right)$ with $\alpha \in \mathbb{D}$. The quotient module, which is the restriction of the original module to the zero set, therefore, consists of functions of the parameter $\alpha$. Since $H^{2}\left(\mathbb{D}^{2}\right)$ is spanned by the collection of monomials $\left\{z_{1}^{k} z_{2}^{\ell}: k, \ell \geq 0\right\}$, it follows that $\mathcal{M}_{\mathrm{q}}$ is spanned by the set $\left\{\alpha^{2 k+3 \ell}: k, \ell \geq 0\right\}$. That is, $\mathcal{M}_{\mathrm{q}}$ is spanned by the set $\left\{\alpha^{n}: n \geq 0, n \neq 1\right\}$ The module action is also defined by restriction and is given by

$$
p \in \mathcal{A}\left(\mathbb{D}^{2}\right) \mapsto p\left(M^{2}, M^{3}\right)
$$

where $M$ denotes multiplication by the coordinate $\alpha$. It follows that the quotient module is indeed isomorphic to the module $H_{1}^{2}(\mathbb{D})$ discussed in Example 2.1. 
EXAMPLE 3.3. We discuss an example where the submodule of functions vanish to a higher order. Now take $\mathcal{M}_{0}$ to be the largest collection of functions in $\mathcal{M}=H^{2}\left(\mathbb{D}^{2}\right)$ which vanish to order 2 on the diagonal $z_{1}=z_{2}$. (See [11] for the details of this example). In this case the quotient module is spanned by the homogeneous polynomials $\left\{e_{k}\right\}$ and $\left\{f_{k}\right\}$, with the $e_{k}$ 's as in Example 3.1 and $f_{k}$ 's defined by

$$
\begin{aligned}
f_{2 m} & =\frac{1}{\alpha_{m}} \sum_{j=0}^{m-1}(m-j) z_{1}^{2 m-j} z_{2}^{j}-\sum_{j=1}^{m} j z_{1}^{m-j} z_{2}^{m+j}, m \geq 1 \\
f_{2 m+1} & =\frac{1}{\beta_{m}} \sum_{j=0}^{m-1}(2 m+1-2 j) z_{1}^{2 m+1-j} z_{2}^{j}-\sum_{j=0}^{m}(2 j+1) z_{1}^{m-j} z_{2}^{m+j+1}, m \geq 1
\end{aligned}
$$

with

$$
\begin{aligned}
\alpha_{m}^{2} & =\frac{1}{3} m(m+1)(2 m+1) \\
\beta_{m}^{2} & =\frac{2}{3}(m+1)(2 m+1)(2 m+3) .
\end{aligned}
$$

The module action is described by

$$
\begin{aligned}
z_{1} \cdot e_{k} & =\sqrt{\frac{k+1}{k+2} e_{k+1}} \\
z_{1} \cdot f_{k} & =\sqrt{\frac{3}{(k+2)(k+3)}} e_{k+1}+\sqrt{\frac{k}{k+3}} f_{k+1} .
\end{aligned}
$$

The action of $z_{2}$ is similar except that the coefficient of $e_{k+1}$ in the second equation above now has a negative sign.

Recall that the quotient module, in the general picture described above, is characterised as a module of vector-valued functions on the zero set, which is one dimensional in this case. The correspondence between this picture and the description in terms of homogeneous polynomials on $\mathbb{D}^{2}$ is obtained via the map

$$
e_{k} \mapsto\left(\begin{array}{c}
\sqrt{k+1} z^{k} \\
\frac{k}{2} \sqrt{k+1} z^{k-1}
\end{array}\right), f_{k} \mapsto\left(\begin{array}{c}
0 \\
\sqrt{\frac{k(k+1)(k+2)}{12}} z^{k-1}
\end{array}\right) .
$$

The matrix-valued reproducing kernel $(J K)_{\text {res }}$ is given by (cf. [14, Lemma 2.2])

$$
(J K)_{\mathrm{res}}(z, w)=\frac{1}{(1-z \bar{w})^{2}}\left(\begin{array}{cc}
1 & z(1-z \bar{w})^{-1} \\
\bar{w}(1-z \bar{w})^{-1} & (1+z \bar{w})(1-z \bar{w})^{-2}
\end{array}\right) .
$$

The complex geometric approach, developed in [6], is applicable to Hilbert modules which give rise to holomorphic bundles where the module action is scalar on each fiber. If two such bundles $\mathcal{E}$ and ${ }^{\sim} E$ are equivalent via the isometric bundle map $\Theta: \mathcal{E} \rightarrow \sim \mathcal{L}$, then it is shown that there exists a unitary $U_{\Theta}: \mathcal{M} \rightarrow \widetilde{\mathcal{M}}$ which implements $\Theta$. Since the action of $\mathcal{A}(\Omega)$ in that treatment is scalar on each 
fiber of the respective bundles and $\Theta$ is a bundle map, a unitary module map is obtained. Although the quotient module in the case of multiplicity $k$ gives rise to a rank $k$ bundle, the action of the algebra $\mathcal{A}(\Omega)$ is no longer scalar on the fiber. Hence, even if we obtain a unitary map $U: \mathcal{M}_{\mathrm{q}} \rightarrow \widetilde{\mathcal{M}}_{\mathrm{q}}$ using techniques from [6], we have to ensure further that this is a module map. Necessary and sufficient conditions for this to happen have not yet been obtained. (In a previous paper [12], it was assumed that $\mathcal{M}_{0}$ is the submodule consisting of all functions vanishing on a hyper-surface $\mathcal{Z}$. In that case, the quotient module gives rise to a line bundle on $\mathcal{Z}$ and the module action is scalar on each fiber. Hence the complex geometric approach of [6] applies.)

The methods described in [6], at least under certain conditions, provide a first step towards a solution. It is hoped that the ideas there can be augmented to provide a complete solution. The general approach is to look at the largest subalgebra of $\mathcal{A}(\Omega)$ which acts by scalars. Equivalence under this action can be examined by the results in [6]. The attempt is then to ascertain when the equivalence can be implemented via an equivalence with respect to the action of the full algebra.

There are many situations in which the quotient modules (as modules over $\mathcal{A}(\mathcal{Z})$ ) lie in $B_{k}(\mathcal{Z})$. (cf. [15, Example 5.16, p. 95] for the definition of this class and the discussion in subsection 2.1.) In these situations, we have the following possible approach to the equivalence question:

If two quotient modules are equivalent, they must be equivalent as modules over the sub-algebra $\mathcal{A}(\mathcal{Z})$. The latter then becomes a question of equivalence in $B_{k}(\mathcal{Z})$. This question has been studied in [6]. For a complete answer to the equivalence question, we need to determine when there is, among all the unitaries that implement the equivalence in $B_{k}(\mathcal{Z})$, one that intertwines the (nilpotent) action of functions depending only on the 'normal' coordinate. This question can be studied in a series of steps as follows:

Notice, firstly, that the action of $z_{1}^{p}$ is given by a $(k-p)$-step nilpotent operator. The requirement that the unitary which describes the $B_{k}(\mathcal{Z})$ equivalence must intertwine these powers of $z_{1}$ translates into a sequence of conditions on the unitary. (For instance, in the case $k=2$, where only the first power of $z_{1}$ is relevant, this requires that the unitary is upper triangular with equal entries on the diagonal. This condition, it must be emphasized, may not ensure that the unitary must also necessarily intertwine the nilpotent elements.)

We are thus led to the following vector bundle picture. If the quotient module lies in $B_{k}(\mathcal{Z})$, there is naturally associated a (rank $k$ ) bundle on $\mathcal{Z}$. However, this bundle now comes equipped with a collection of sub-bundles which together determine a flag on each fiber. The full equivalence of the quotient modules requires, among other things, the equivalence of these 'flag bundles'. We point out that equivalence of these flags will determine the equivalence of the modules upto "similarity". We will need the metric structure to obtain unitary invariants.

Equivalence of flag bundles, at least formally like these, is considered and characterized by Martin and Salinas [20, Theorem 4.5]. 
The quotient module $\mathcal{M}_{\mathrm{q}}$ is described in [14]. The modules $\mathcal{M}, \mathcal{M}_{0}$ and $\mathcal{M}_{\mathrm{q}}$ are identified with geometric objects, specifically vector bundles, though not all on the same base. The equivalence of quotient modules is then reduced to a suitable equivalence of these geometric objects.

The program will be complete only if one now obtains (hopefully complete) invariants for these geometric objects. Towards this end the work of Bott and Chern [3] and after them, Bismut, Gillet and Soulé [2], is probably relevant. These authors study invariants for chain complexes of vector bundles on a given base. However, it seems plausible that their ideas can be extended to chain complexes of more general geometric objects of the type encountered in the study of quotient modules. If these ideas can be carried to their logical end, we will have a rich geometric complement to the module theoretic results obtained so far. In fact our results produce, in many cases, natural examples to which the results of [3] as well as [19] apply.

A second line of enquiry in the geometric direction is a possible adaptation of the results of Harvey and Lawson [19] to the present situation. Let $\mathcal{E}$ and $\mathcal{E}_{0}$ be the vector bundles obtained, as mentioned above, by localisation, from the modules $\mathcal{M}$ and $\mathcal{M}_{0}$ and let $\phi$ be an ad-invariant polynomial (in particular, a Chern form) in the respective curvatures $\mathcal{K}$ and $\mathcal{K}_{0}$. Then the work of Harvey and Lawson [19] on singular connections gives a mechanism for studying these bundles since the natural connection on the bundle $\mathcal{M}_{0}$ is a singular one. They obtain a relation of the form

$$
\phi(\mathcal{K})-\phi\left(\mathcal{K}_{0}\right)=\operatorname{Res}_{\phi}[\mathcal{Z}]+d T_{\phi}
$$

where $\operatorname{Re} s_{\phi}[\mathcal{Z}]$ is a 'residue' form related to the zero set and $T_{\phi}$ is a transgression current. Note that this incorporates a generalised Poincaré-Lelong formula which played a crucial role in the study of the quotient module in the rank one case [12].

By comparison with the rank one case, it seems likely that the current $T_{\phi}$ is directly related to the map from $\mathcal{E}_{0}$ to $\mathcal{E}$ induced by the inclusion map from $\mathcal{M}_{0}$ into $\mathcal{M}$. By studying the collection $\{\operatorname{Res} \phi[\mathcal{Z}]\}$ for various choices of the the adinvariant polynomial $\phi$, it is hoped that a complete characterisation of the quotient module can be obtained.

\section{The case of ideals which are not necessarily principal}

The approaches outlined above have been in the context where $\mathcal{M}_{0}$ consisted of functions which vanished to some order on the hypersurface $\mathcal{Z}$. In most cases, $\mathcal{M}_{0}$ is the closure of a principal ideal in $\mathcal{M}$. The problem changes character completely if we allow $\mathcal{M}_{0}$ to be the closure of an ideal which is not necessarily principal.

Suppose that the zero variety $\mathcal{Z} \subseteq \Omega$ is a hypersurface and $\mathcal{M}_{0}$ is the maximal set of functions in $\mathcal{M}$ which vanish on $\mathcal{Z}$. Then using the Poincaré-Lelong formula, 
it follows that the alternating sum

$$
\sum_{i, j=1}^{m} \frac{\partial^{2}}{\partial w_{i} \partial \bar{w}_{j}} \log \left(X(w)^{*} X(w)\right) d w_{i} \wedge d \bar{w}_{j}-\mathcal{K}_{0}(w)+\mathcal{K}(w)
$$

represents the fundamental class $[\mathcal{Z}]$, where $\mathcal{K}$ and $\mathcal{K}_{0}$ are the curvatures associated with the modules $\mathcal{M}$ and $\mathcal{M}_{0}$ respectively and $X(w)$ is the localisation of the map $X$ at $w \in \Omega$.

If the zero variety is not a hypersurface, there are at least two different versions of the Poincaré-Lelong formula. In each of these cases, the current of integration on $\mathcal{Z}$ is identified with a current on $\Omega$ of bi-degree $(m-r, m-r)$, where $r$ is the codimension of the variety $\mathcal{Z}$. If $\mathcal{M}_{0}$ is the maximal set of functions in $\mathcal{M}$ which vanish on $\mathcal{Z}$, then we construct two different resolutions of the quotient module and find that they lead in a natural manner to the current of integration. Of course, this current is an unitary invariant for the quotient module.

As mentioned in the introduction, the complex

$$
0 \longrightarrow \mathcal{M} \stackrel{M_{\varphi}}{\longrightarrow} \mathcal{M} \longrightarrow 0
$$

is the simplest example of a Koszul complex (discussed in the next section) and the sequence (1.3) is an extension of this Koszul complex by the quotient module.

We therefore consider the following natural extension when $\mathcal{M}_{0}$ is the set of functions vanishing on an analytic submanifold of higher codimension. If $\mathcal{Z}$ is defined by the vanishing of the functions $\varphi_{1}, \varphi_{2}, \cdots, \varphi_{r}$, we consider the multiplication operators $M_{\varphi_{1}}, M_{\varphi_{2}}, \cdots, M_{\varphi_{r}}$ and the associated Koszul complex (extended by the quotient module):

$$
0 \longrightarrow \mathbb{H}_{0} \stackrel{d_{0}}{\longrightarrow} \mathbb{H}_{1} \stackrel{d_{1}}{\longrightarrow} \mathbb{H}_{2} \stackrel{d_{2}}{\longrightarrow} \cdots \stackrel{d_{r-1}}{\longrightarrow} \mathbb{H}_{r} \longrightarrow \mathcal{M}_{\mathrm{q}} \longrightarrow 0
$$

We then attempt to construct invariants for the quotient module making use of the differential maps $d_{0}, d_{1}, d_{2}, \cdots, d_{r-1}$ of the complex. As a first step, we recover the fundamental class of $\mathcal{Z}$ using the differential maps and the application of a generalised Poincaré-Lelong formula.

It must be pointed out that although in [13] and [14] the approach has been to use a resolution of the quotient module by means of an exact sequence, we make a departure here. Our focus here has been enlarged to chain complexes which terminate in the quotient module and which may not be necessarily exact. This has been motivated by the results in [13] where it is shown that it is possible to construct invariants for the quotient module by considering complexes which are not necessarily exact.

We further assume that $\mathcal{Z}$ is an analytic submanifold of $\Omega$ defined by the vanishing of functions $\varphi_{1}, \varphi_{2}, \cdots, \varphi_{r}$ defined globally on $\Omega$. These functions are assumed to be holomorphic on $\Omega$ and continuous on $\bar{\Omega}$. The module $\mathcal{M}_{0}$ is then taken to be the submodule of all functions in $\mathcal{M}$ which vanish on $\mathcal{Z}$ and $\mathcal{M}_{\mathrm{q}}$ is defined to be the quotient module. 


\subsection{The Koszul Complex}

The invariant that we propose to construct uses the Koszul complex for a prescribed set of operators. We, therefore, outline the construction of the Koszul complex for an $r$-tuple of operators on a Hilbert space. A useful reference here is [16].

Let $\mathcal{H}$ be a Hilbert space and let $T_{1}, T_{2}, \cdots, T_{r}$ be a commuting $r$-tuple of operators on $\mathcal{H}$. Let $\wedge(\epsilon)$ be the (graded) exterior algebra on $r$ symbols $\epsilon_{1}, \epsilon_{2}, \cdots, \epsilon_{r}$. Denote by $\wedge^{j}(\epsilon)$ the exterior space spanned by the set $\left\{\epsilon_{l_{1}} \wedge \epsilon_{l_{2}} \wedge \cdots \wedge \epsilon_{l_{j}}\right\}$ for $j=0,1, \ldots, r$. Let

$$
\mathbb{H}_{j}=\mathcal{H} \otimes \wedge^{j}(\epsilon) .
$$

Define the differential $d_{j}: \mathbb{H}_{j} \rightarrow \mathbb{H}_{j+1}$ by $d_{j}=\sum_{i=1}^{r} T_{i} \otimes \sigma_{i}$ where $\sigma_{i}$ is the operator defined by

$$
\sigma_{i}(\xi)=\epsilon_{i} \wedge \xi, \xi \in \wedge(\epsilon) .
$$

The adjoint $\sigma_{i}^{*}$ of $\sigma_{i}$ is given by

$$
\sigma_{i}^{*}\left(\xi_{1}+\epsilon_{i} \wedge \xi_{2}\right)=\xi_{2}
$$

where $\xi_{1}$ and $\xi_{2}$ do not contain the symbol $\epsilon_{i}$. We therefore have the following:

$$
\begin{aligned}
\sigma_{i} \sigma_{j}+\sigma_{j} \sigma_{i} & =0 \\
\sigma_{i} \sigma_{j}^{*}+\sigma_{j}^{*} \sigma_{i} & =\delta_{i j} \text { for } i, j=1,2, \ldots, r,
\end{aligned}
$$

where $\delta$ is the Kronecker symbol. It follows that $d_{j+1} d_{j}=0$.

Define the Laplace operator on $\mathbb{H}_{j}$ by

$$
\Delta_{j}=d_{j}^{*} d_{j}+d_{j-1} d_{j-1}^{*} \text { for } j=1,2, \ldots, r-1 .
$$

Also take $\Delta_{0}=d_{0}^{*} d_{0}$ and $\Delta_{r}=d_{r-1} d_{r-1}^{*}$.

The operator $d_{j}^{*}$ acts on $\mathbb{H}_{j+1}$ as the adjoint of $d_{j}$ and is given by

$$
d_{j}^{*}=\sum_{i=1}^{r} T_{i}^{*} \otimes \sigma_{i}^{*} .
$$

The torsion, $\tau$, of the Koszul complex, when $\mathcal{H}$ is finite dimensional, is defined through the expression

$$
\log \tau=\sum_{j=0}^{r}(-1)^{j} j \log \operatorname{det} \Delta_{j} .
$$

Consider the case where $T_{1}, T_{2}, \cdots, T_{r}$ represent a family of operators which depend holomorphically on a parameter $w \in \Omega \subseteq \mathbb{C}^{m}$. This parametrized family of maps, $T_{1}, T_{2}, \cdots, T_{r}$, gives rise to a parametrized family of Koszul complexes, as above. Motivated by the definition of the torsion, we make the following formal definition which associates, under certain circumstances, an $(r-1, r-1)$ current to such a parametrized family of Koszul complexes. We note that $\operatorname{det} \Delta_{j}(w)$ is real analytic, and consequently, each term in the product below is a current. However, this does not ensure that the product is a current, in general. The results on page 161 of [17] show that the product is indeed a current in many cases. However, in 
our case, the hypothesis that the common zero set is a complex analytic manifold ensures the transversality of the normal directions. Hence the singular wave front sets for the factors are disjoint and the product is well defined as a current. We also point out that for the parametrized family that we obtain from the localisation of the resolution of the quotient module, this definition yields the current

$$
\Theta=\frac{1}{\alpha}\left(\log \operatorname{det}\left(\Delta_{0}(w)\right) \prod_{j=1}^{r-1} \partial \bar{\partial} \log \operatorname{det}\left(\Delta_{j}(w)\right),\right.
$$

where $\alpha$ is the numerical constant given by $\alpha=\prod_{j=0}^{r-1}\left(\begin{array}{c}r \\ j\end{array}\right)$.

LEMma 4.1. Let $T_{1}, T_{2}, \cdots, T_{r}$ be a commuting tuple of operators as above. If $\left[T_{i}, T_{j}^{*}\right]=0$ for $i, j=1,2, \ldots, r$, then $\Delta_{j}$ as introduced above is given by

$$
\Delta_{j}=\left(\sum_{i=1}^{r} T_{i} T_{i}^{*}\right) \otimes 1_{j},
$$

where $1_{j}$ is the identity operator on $\wedge^{j}(\epsilon)$.

Moreover, in this case, if $\mathcal{H}$ is finite dimensional, we have the following:

(i) $\operatorname{Tr} \Delta_{j}=\left(\begin{array}{l}r \\ j\end{array}\right) \operatorname{Tr}\left(\sum_{i=1}^{r} T_{i} T_{i}^{*}\right)$ where $\operatorname{Tr}$ denotes the trace.

(ii) $\log \operatorname{det} \Delta_{j}=\left(\begin{array}{c}r \\ j\end{array}\right) \log \operatorname{det}\left(\sum_{i=1}^{r} T_{i} T_{i}^{*}\right)$.

(iii) The torsion $\tau=1$.

Proof: The first result follows from the definition of $\Delta_{j}$ using (4.3). The others follow from the fact that the dimension of $\wedge^{j}(\epsilon)$ is $\left(\begin{array}{c}r \\ j\end{array}\right)$.

The fundamental class of $\mathcal{Z}$ is recovered by an application of the following generalised Poincaré-Lelong formula. For a proof and more details see [17].

THEOREM 4.1. Let $\Omega$ be a complex $n$-manifold and let

$$
\varphi_{1}, \varphi_{2}, \cdots, \varphi_{r}: \Omega \rightarrow \mathbb{C}
$$

be holomorphic maps. Let $\mathcal{Z}$ be the common zero set of $\varphi_{1}, \varphi_{2}, \cdots, \varphi_{r}$. Let

$$
\Theta=\log \|\varphi\|^{2}\left(\partial \bar{\partial} \log \|\varphi\|^{2}\right)^{r-1},
$$

where $\|\varphi\|^{2}=\left|\varphi_{1}\right|^{2}+\left|\varphi_{2}\right|^{2}+\cdots+\left|\varphi_{r}\right|^{2}$.

If $\mathcal{Z}$ has dimension $n-r, \partial \bar{\partial} \Theta$ defines a current of degree $(r, r)$ and corresponds to the fundamental class of $\mathcal{Z}$. In symbols, if $\omega$ is a compactly supported $(n-r, n-r)$ form, then

$$
\int_{\Omega} \Theta \wedge \partial \bar{\partial} \omega=\int_{\mathcal{Z}} \omega
$$




\subsection{Invariants for the Quotient Module}

We now have the necessary ingredients to recover the fundamental class of the zero set $\mathcal{Z}$. If $\mathcal{Z}$ is defined by the vanishing of $\varphi_{1}, \varphi_{2}, \cdots, \varphi_{r}$, let $M_{\varphi_{1}}, M_{\varphi_{2}}, \cdots, M_{\varphi_{r}}$ be the operators of multiplication by $\varphi_{1}, \varphi_{2}, \cdots, \varphi_{r}$. Construct the (extended) Koszul complex corresponding to the commuting operators $M_{\varphi_{1}}, M_{\varphi_{2}}, \cdots, M_{\varphi_{r}}$ :

$$
0 \longrightarrow \mathcal{M} \otimes \wedge^{0}(\epsilon) \stackrel{d_{0}}{\longrightarrow} \mathcal{M} \otimes \wedge^{1}(\epsilon) \stackrel{d_{1}}{\longrightarrow} \cdots \stackrel{d_{r-1}}{\longrightarrow} \mathcal{M} \otimes \wedge^{r}(\epsilon) \longrightarrow \mathcal{M}_{\mathrm{q}} \longrightarrow 0
$$

Since $\mathcal{M} \otimes \wedge^{r}(\epsilon)$ is isomorphic to $\mathcal{M}$, the map at the last stage is taken to be the quotient map. We can make $\mathcal{M} \otimes \wedge^{j}(\epsilon)$ a module over $\mathcal{A}$ by choosing the trivial action on the second factor. With this choice of action, it follows that

$$
\left(\mathcal{M} \otimes \wedge^{j}(\epsilon)\right) \otimes_{\mathcal{A}} \mathbb{C}_{w}=\left(\mathcal{M} \otimes_{\mathcal{A}} \mathbb{C}_{w}\right) \otimes \wedge^{j}(\epsilon)
$$

With the assumptions we have made on $\mathcal{M}$, it can be shown that $\mathcal{M} \otimes_{\mathcal{A}} \mathbb{C}_{w}$ is one dimensional. Therefore, $\operatorname{dim}\left(\left(\mathcal{M} \otimes \wedge^{j}(\epsilon)\right) \otimes_{\mathcal{A}} \mathbb{C}_{w}\right)=\left(\begin{array}{l}r \\ j\end{array}\right)$.

THEOREM 4.2. Consider the localisation of the above complex (4.6) by the module $\mathbb{C}_{w}, w \in \Omega$. Let $\Theta$ be the $(r-1, r-1)$ current defined in equation (4.4), now applied to this complex. Then $\partial \bar{\partial} \Theta$ represents the fundamental class of $\mathcal{Z}$.

Proof: Since $\varphi_{1}, \ldots, \varphi_{r} \in \mathcal{A}$, it follows that the two actions $M_{\varphi_{j}} \otimes I$ and $I \otimes M_{\varphi_{j}(w)}$ are identified on $\mathcal{M} \otimes_{\mathcal{A}} \mathbb{C}_{w}$. Consequently the localisation of $M_{\varphi_{j}}$, denoted $M_{\varphi_{j}}(w)$, is the same as the one dimensional operator $M_{\varphi_{j}(w)}$. Hence localising the above extended Koszul complex is equivalent to constructing the Koszul complex for the operators $M_{\varphi_{1}(w)}, \ldots, M_{\varphi_{r}(w)}$ (each of which acts on the one dimensional Hilbert space $\left.\mathcal{H}=\mathcal{M} \otimes_{\mathcal{A}} \mathbb{C}_{w}\right)$ and then extending the complex by $\mathcal{M}_{\mathrm{q}} \otimes_{\mathcal{A}} \mathbb{C}_{w}$.

The operator $\sum_{i=1}^{r} T_{i} T_{i}^{*}$ occuring in Lemma 4.1 is the (parametrized) one dimensional operator represented by $\left|\varphi_{1}(w)\right|^{2}+\left|\varphi_{2}(w)\right|^{2}+\cdots+\left|\varphi_{r}(w)\right|^{2}$. Now, the result follows from an application of this Lemma and Theorem 4.1.

REMARK 4.1. Now consider just the Koszul complex for the operators $M_{\varphi_{1}}, \ldots, M_{\varphi_{r}}$ :

$$
0 \longrightarrow \mathcal{M} \otimes \wedge^{0}(\epsilon) \stackrel{d_{0}}{\longrightarrow} \mathcal{M} \otimes \wedge^{1}(\epsilon) \stackrel{d_{1}}{\longrightarrow} \cdots \stackrel{d_{r-1}}{\longrightarrow} \mathcal{M} \otimes \wedge^{r}(\epsilon) \longrightarrow 0 .
$$

It would be natural to ask if the torsion of the localisation of this complex is an invariant for the quotient module. If the localisation is done with the one dimensional module $\mathbb{C}_{w}$, it is clear that the torsion is trivial using Lemma 4.1. We could localise using higher rank modules. Lemma 4.1 would no longer be relevant (since $T_{i}$ and $T_{j}^{*}$ may not commute). However, we have noticed that, in some cases where the torsion can be computed explicitly, it still turns out be trivial. This is true, for instance, if we localize with the two dimensional module $\mathbb{C}_{w}^{2}$ using the module action described in [14]. Whether this is true in some generality is an interesting question.

Another resolution of the quotient module is obtained as a grid of short exact sequences of Hilbert modules in which each line consists of a short exact sequence. We illustrate our methods in the case where the the zero variety is a complete 
intersection of two hypersurfaces $\mathcal{Z}_{1}$ and $\mathcal{Z}_{2}$. Let $\mathcal{I}_{1}$ and $\mathcal{I}_{2}$ be the maximal set of functions which vanish on the hypersurfaces $\mathcal{Z}_{1}$ and $\mathcal{Z}_{2}$ respectively and let $\mathcal{I}_{12}=\mathcal{I}_{1} \cap \mathcal{I}_{2}$. Let us consider the diagram

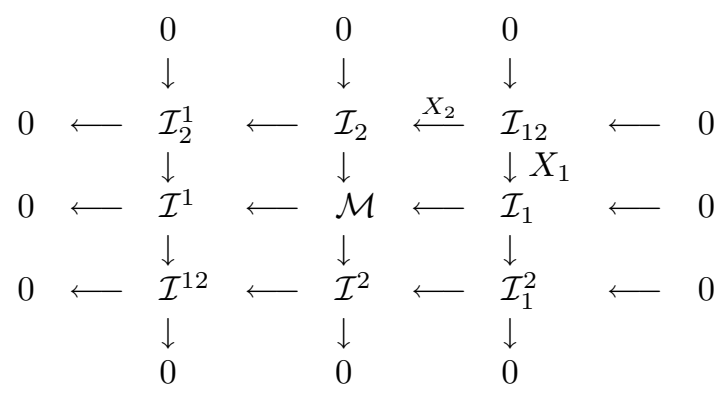

where each connecting map is either an inclusion or a quotient map. We require that each row and column be a short exact sequence of Hilbert modules. Hence all the undefined symbols in the above diagram represent quotient modules at intermediate stages. Localisation of the grid above provides the necessary ingredients to obtain the current of integration on the zero variety $\mathcal{Z}_{1} \cap \mathcal{Z}_{2}$, using the following theorem, which is a generalisation of the Poincaré-Lelong equation (cf. [8]). It is the case $p=2$ of the theorem which is relevant to the above grid.

THEOREM 4.3. Let $\varphi=\left(\varphi_{1}, \cdots, \varphi_{p}\right): U \rightarrow \mathbb{C}^{p}$ be a holomorphic mapping. If $Y_{j}=\varphi_{j}^{-1}\{0\}$ are the loci of zeros of the components of $\varphi$, one has

$$
\bar{\partial} \partial \log \left|\varphi_{1}\right|^{2} \wedge \cdots \wedge \bar{\partial} \partial \log \left|\varphi_{p}\right|^{2}=2 \pi i\left[Y_{1}\right] \wedge \cdots \wedge 2 \pi i\left[Y_{p}\right]=(2 \pi i)^{p}[Y]
$$

where $[Y]$ is the current corresponding to the intersection $Y_{1} \cap \cdots \cap Y_{p}$.

Notice that $\mathcal{I}^{12}$ in the grid above is the quotient module we are attempting to describe. The result above is then a natural generalisation of the approach described following the linear resolution (1.1) of the quotient module. Specifically (see discussion in subsection 2.4),

$$
X_{\ell}(w)^{*} X_{\ell}(w)=\frac{\left|\varphi_{1}(w) \varphi_{2}(w)\right|^{2} \chi_{12}(w, w)}{\left|\varphi_{\ell}(w)\right|^{2} \chi_{\ell}(w, w)}, \ell=1,2 .
$$

Consequently, we find that

$$
\begin{array}{r}
\sum_{i, j=1}^{m} \frac{\partial^{2}}{\partial w_{i} \partial \bar{w}_{j}} \log \left(X_{\ell}(w)^{*} X_{\ell}(w)\right) d w_{i} \wedge d \bar{w}_{j}-\mathcal{K}_{12}(w)+\mathcal{K}_{\ell}(w) \\
=\sum_{i, j=1}^{m} \frac{\partial^{2}}{\partial w_{i} \partial \bar{w}_{j}} \log \left|\varphi_{\ell}(w)\right|^{2} d w_{i} \wedge d \bar{w}_{j},
\end{array}
$$

for $\ell=1,2$. Hence Theorem 4.3 applies and we conclude that the product of the two currents described in (4.10) yield the fundamental class of the zero variety. 
Even though the grid above is not a resolution of the quotient module in the usual sense, we can extract a linear exact sequence which is a resolution of the quotient. This resolution is obtained from the grid as follows.

We reproduce here the general technique of obtaining a linear complex from a two dimensional complex and then apply the procedure to the case at hand. Let

$$
\begin{aligned}
C_{i, j+1} \stackrel{\delta_{i, j}}{\longleftarrow} & C_{i, j} \\
& d_{i, j} \downarrow \\
& C_{i+1, j}
\end{aligned}
$$

be a planar grid for $0 \leq i, j \leq N$, where $d^{2}$ and $\delta^{2}$ are zero. This grid gives rise to a linear complex in a natural manner as follows. The $k$-th term of the linear complex is

$$
D_{k}=\bigoplus_{i+j=k} C_{i, j}
$$

The boundary maps $\Delta_{k}: D_{k} \rightarrow D_{k+1}$ are defined by

$$
\left.\Delta_{k}\right|_{C_{i, j}}=\delta_{i, j}+(-1)^{j} d_{i, j}
$$

We can apply this to the planar grid of modules above. Recall that $\mathcal{I}_{k}, k=1,2$ are the ideals of functions vanishing on the hypersurface $\mathcal{Z}_{k}=\left\{z \in \Omega: \varphi_{k}(z)=0\right\}$ and that $\mathcal{I}_{12}$ is the intersection of these two ideals. This leads to the following exact sequence.

$0 \leftarrow Q \stackrel{\Delta_{4}}{\longleftarrow}\left(\mathcal{M} / \mathcal{I}_{1}\right) \oplus\left(\mathcal{M} / \mathcal{I}_{2}\right) \stackrel{\Delta_{3}}{\longleftarrow}\left(\mathcal{I}_{1} / \mathcal{I}_{12}\right) \oplus \mathcal{M} \oplus\left(\mathcal{I}_{2} / \mathcal{I}_{12}\right) \stackrel{\Delta_{2}}{\longleftarrow} \mathcal{I}_{1} \oplus \mathcal{I}_{2} \stackrel{\Delta_{1}}{\longleftarrow} \mathcal{I}_{12} \leftarrow 0$

The maps $\Delta_{k}$ are defined by

$$
\begin{aligned}
\Delta_{1}(x) & =(x, x) \\
\Delta_{2}(x, y) & =\left(P_{12}^{1} x,-x+y, P_{12}^{2} y\right) \\
\Delta_{3}(x, y, z) & =\left(-P_{1} y+z, x+P_{2} y\right) \\
\Delta_{4}(x, y) & =P_{11} x+P_{22} y,
\end{aligned}
$$

where

a) $P_{12}^{1}$ (respectively $P_{12}^{2}$ ) is the quotient map from $\mathcal{I}_{1}$ (respectively $\mathcal{I}_{2}$ ) to $\mathcal{I}_{1} / \mathcal{I}_{12}$ (respectively $\mathcal{I}_{2} / \mathcal{I}_{12}$ ),

b) $P_{1}$ (respectively $P_{2}$ ) is the quotient map from $\mathcal{M}$ to $\mathcal{M} / \mathcal{I}_{1}$ (respectively $\mathcal{M} / \mathcal{I}_{2}$ ), c) $P_{11}$ (respectively $P_{22}$ ) is the relevant quotient map that appears in the grid from $\mathcal{M} / \mathcal{I}_{1}$ (respectively $\left.\mathcal{M} / \mathcal{I}_{2}\right)$ ) to the quotient module $(Q)$.

We point out here that there is a generalisation of the planar grid to higher dimensions which becomes relevant when the codimension of the zero set is greater than two. In fact, we then need to consider a hypercubic grid in as many dimensions as the codimension. Theorem 4.3 can be used in this general case to once again determine the current of integration on the zero set. The linearisation described above also has a natural generalisation. 


\section{Localisation techniques and invariants for submodules}

In this section, we use the localisation technique to describe some possible invariants for submodules rather than quotient modules.

Let $\mathcal{I}$ be an ideal in the polynomial ring $\mathbb{C}\left[z_{1}, \cdots, z_{m}\right]$. Let $\mathcal{M}_{0} \subseteq \mathcal{M}$ be the submodule which is the closure of such an ideal. The submodule $\mathcal{M}_{0}$ is therefore contained in the largest set of functions in $\mathcal{M}$ which vanish on the common zero set of this ideal - we assume that this inclusion is indeed an equality. It is easy to see that the localisation (cf. [15]) of the submodule $\mathcal{M}_{0}$ at $w=\left(w_{1}, \cdots, w_{m}\right) \in \Omega$ is $\cap_{\ell=1}^{m} \operatorname{ker}\left(M_{z_{\ell}} \mid \mathcal{M}_{0}-w_{\ell}\right)^{*} \subseteq \mathcal{M}_{0}$. These subspaces inherit a hermitian metric from the module $\mathcal{M}_{0}$. In case the submodule is the closure of an ideal generated by a set of polynomials, we conjecture that

$$
\operatorname{dim} \cap_{\ell=1}^{m} \operatorname{ker}\left(\left.M_{z_{\ell}}\right|_{\mathcal{M}_{0}}-w_{\ell}\right)^{*}= \begin{cases}1 & \text { for } w \notin V(\mathcal{I}) \\ \text { codimension of } V(\mathcal{I}) & \text { for } w \in V(\mathcal{I})\end{cases}
$$

where $V(\mathcal{I})$ is the common zero set of the ideal $\mathcal{I}$ intersect $\Omega$. It is clear that in case $\mathcal{Z}=V(\mathcal{I})$ is a hypersurface, the localisation produces a holomorphic hermitian line bundle. Consequently, the complex geometric approach developed in [6] gurantee that the curvature of this bundle is a complete invariant for the submodule $\mathcal{M}_{0}$. However, if $\mathcal{Z}$ is not a hypersurface, the dimension of the localisation jumps across the zero set.

If $K$ is the kernel function for $\mathcal{M}$, then $K(\cdot, w)$ induces a map $\gamma: \Omega \rightarrow \mathcal{M}^{*}$ via $\gamma(w)(f)=\langle f, K(\cdot, w)\rangle, f \in \mathcal{M}$. Since the constant functions are in $\mathcal{M}$, it follows that $\gamma(w) \neq 0, w \in \Omega$. Thus $\gamma$ maps into the projective space $\operatorname{Gr}(\mathcal{M}, 1)$. The bundle described in the preceding paragraph is the pull back of the canonical bundle on the projective space under this map. However, if we attempt a similar approach with $\mathcal{M}_{0}$, then we find that we must consider the map induced by the vectors $P K(\cdot, w)$, where $P$ is the projection to $\mathcal{M}_{0}$ :

The localisation $\mathcal{M}_{0}(w)=\mathcal{M}_{0} \otimes_{\mathcal{A}} \mathbb{C}_{w}$ is the orthogonal complement (in $\left.\mathcal{M}_{0}\right)$ of the subspace

$$
\begin{aligned}
\mathcal{N}_{w} & =\operatorname{span}\left\{(f \cdot h) \otimes 1-h \otimes(f \cdot 1): f \in \mathcal{A}(\Omega), h \in \mathcal{M}_{0}\right\} \\
& =\operatorname{span}\left\{((f-f(w)) \cdot h) \otimes 1: f \in \mathcal{A}(\Omega), h \in \mathcal{M}_{0}\right\}
\end{aligned}
$$

We clearly have $\mathcal{N}_{w} \subseteq\left\{h \in \mathcal{M}_{0}: h(w)=0\right\}$ for $w \notin \mathcal{Z}$. Since $\mathcal{M}_{0}$ is the closure of an ideal in $\mathbb{C}\left[z_{1}, \ldots, z_{m}\right] \subseteq \mathcal{M}$, it is likely that $\mathcal{N}_{w}=\left\{h \in \mathcal{M}_{0}: h(w)=0\right\}, w \notin \mathcal{Z}$. Since $\mathcal{N}_{w} \subseteq \mathcal{M}_{0} \subseteq \mathcal{M}$, it follows that as long as $w \notin \mathcal{Z}$, and $\mathcal{N}_{w} \subseteq \mathcal{M}_{0}$ is the submodule of functions vanishing at $w$, we have

$$
\begin{aligned}
\mathcal{M}_{0} \ominus \mathcal{N}_{w} & =\left(\mathcal{M} \ominus \mathcal{N}_{w}\right) \ominus\left(\mathcal{M} \ominus \mathcal{M}_{0}\right) \\
& =\operatorname{clos} \operatorname{span}\{K(\cdot, w), K(\cdot, \lambda): \lambda \in \mathcal{Z}\} \ominus \operatorname{clos} \operatorname{span}\{K(\cdot, \lambda): \lambda \in \mathcal{Z}\} \\
& =P K(\cdot, w),
\end{aligned}
$$

where $K$ is the reproducing kernel of the module $\mathcal{M}$ and $P$ is the orthogonal projection on to $\mathcal{M}_{0}$. 
Since $K_{0}(\cdot, w)=P K(\cdot, w)$ is zero for $w \in V(\mathcal{I})$, we find that the associated map $\gamma$ vanishes at such points. As was pointed out in Remark 2.4, it is not possible to get around this difficulty in a straightforward manner unless $\mathcal{Z}$ is a hypersurface. Nevertheless, we show how we may take limits of $\operatorname{PK}(\cdot, w)$ along complex lines to get around this difficulty.

EXAMPLE 5.1 (Continuation of Example 1.1). Let $H^{2}\left(\mathbb{D}^{2}\right)$ be the Hardy space on the bi-disc $\mathbb{D}^{2}$ and let

$$
0 \leftarrow \mathcal{M}_{\mathrm{q}} \leftarrow H^{2}\left(\mathbb{D}^{2}\right) \leftarrow H_{0}^{2}\left(\mathbb{D}^{2}\right) \leftarrow 0
$$

be a short exact sequence of modules over the bi-disc algebra. Here, $H_{0}^{2}\left(\mathbb{D}^{2}\right)$ is the ideal generated in $H^{2}\left(\mathbb{D}^{2}\right)$ by the coordinate functions $z_{1}, z_{2}$ of $\mathbb{D}^{2}$. The quotient module in this case is 1 dimensional, the spectrum of $\mathcal{M}_{\mathrm{q}}$ is the singleton set $\{0\}$. The localisation of this module $\mathcal{M}_{\mathrm{q}}$ is the skyscraper sheaf at the origin. The bundle for $H_{0}^{2}\left(\mathbb{D}^{2}\right)$ is only defined over $\mathbb{D}^{2} \backslash\{0\}$. The rank of the fibre (which is 1 on $\mathbb{D}^{2} \backslash\{0\}$ ) jumps to 2 at the origin and hence we get a "hermitian" sheaf on $\mathbb{D}^{2}$ rather than a bundle.

A natural question to ask is how to construct invariants for such a "hermitian" sheaf so that they play the same role as the curvature does for hermitian bundles. For the example at hand, one has the following approach.

Let $w=\left(w_{1}, w_{2}\right) \in \mathbb{D}^{2} \backslash\{0\}$. We will compute the module $H_{0}^{2}\left(\mathbb{D}^{2}\right) \otimes_{\mathcal{A}} \mathbb{C}_{w}$, where $\mathcal{A}$ is the bi-disc algebra consisting of functions which are continuous on the closed bi-disc and are holomorphic on the bi-disc. Since $\left\{z_{1}, z_{2}\right\}$ forms a minimal set of generators for the module $H_{0}^{2}\left(\mathbb{D}^{2}\right)$, the set $\left\{z_{1} \otimes 1_{w}, z_{2} \otimes 1_{w}\right\}$ spans the whole module $H_{0}^{2}\left(\mathbb{D}^{2}\right) \otimes \mathbb{C}_{w}$. The localization $H_{0}^{2}\left(\mathbb{D}^{2}\right) \otimes_{\mathcal{A}} \mathbb{C}_{w}$ is

$$
H_{0}^{2}\left(\mathbb{D}^{2}\right) \ominus\left\{h \in H_{0}^{2}\left(\mathbb{D}^{2}\right): h(w)=0\right\} .
$$

As pointed out earlier this space is spanned by the projection of $\hat{K}(\cdot, w)$, w $=$ $\left(w_{1}, w_{2}\right) \in \mathbb{D}^{2}$, to $\mathcal{M}_{0}$ which equals $\hat{K}(\cdot, w)-\hat{K}(\cdot, 0)=\hat{K}_{0}(\cdot, w)$ defined in Example 1.1. This gives a basis vector for the one dimensional space obtained by localising using $\mathbb{C}_{w}$ for $w \neq 0$.

From the explicit description of $\hat{K}_{0}$, we find that the limit of $\hat{K}_{0}(\cdot, w)$ as $w \rightarrow 0$ is 0 . Nevertheless, the limit of $\frac{1}{\bar{w}_{\ell}} \hat{K}_{0}(\cdot, w)$ along lines through the origin as $w \rightarrow 0$ exist and is non-zero for $\ell=1,2$. If we parametrize the lines through the origin by $\bar{w}_{2}=\theta_{1} \bar{w}_{1}$ or $\bar{w}_{1}=\theta_{2} \bar{w}_{2}$, we get

$$
\begin{aligned}
\lim _{\bar{w}_{2}=\theta_{1} \bar{w}_{1}, w \rightarrow 0} \frac{\hat{K}_{0}(z, w)}{\bar{w}_{1}} & =z_{1}+\theta_{1} z_{2} \\
& =\left.\left(\bar{\partial}_{1}+\theta_{1} \bar{\partial}_{2}\right) \hat{K}_{0}(z, w)\right|_{w=0} \\
\lim _{\bar{w}_{1}=\theta_{2} \bar{w}_{2}, w \rightarrow 0} \frac{\hat{K}_{0}(z, w)}{\bar{w}_{2}} & =\theta_{2} z_{1}+z_{2} \\
& =\left.\left(\theta_{2} \bar{\partial}_{1}+\bar{\partial}_{2}\right) \hat{K}_{0}(z, w)\right|_{w=0} .
\end{aligned}
$$


We now construct a line bundle on the projective space $\mathbb{P}^{1}$ (obtained by "blowing up the origin"). The section $s_{1}$ on the coordinate patch $U=\left\{w \in \mathbb{D}^{2}: w_{1} \neq 0\right\}$ is given (as an element of $H_{0}^{2}\left(\mathbb{D}^{2}\right)$ ) by

$$
\begin{aligned}
\left(s_{1}\left(\theta_{1}\right)\right)(z) & =\left.\left(\bar{\partial}_{1} K_{0}(z, w)\right)\right|_{w=0}+\left.\theta_{1}\left(\bar{\partial}_{2} K_{0}(z, w)\right)\right|_{w=0} \\
& =z_{1}+\theta_{1} z_{2} .
\end{aligned}
$$

The squared norm of this section is $1+\left|\theta_{1}\right|^{2}$. Consequently the curvature of the line bundle on the affine set $\left\{w_{1} \neq 0\right\}$ is given by

$$
\begin{aligned}
\mathcal{K}\left(\theta_{1}\right) & =\partial_{\theta_{1}} \partial_{\bar{\theta}_{1}} \log \frac{1}{1+\left|\theta_{1}\right|^{2}} \\
& =\frac{d \theta_{1} \wedge d \bar{\theta}_{1}}{\left(1+\left|\theta_{1}\right|^{2}\right)^{2}} .
\end{aligned}
$$

Obviously, a similar calculation yields $\mathcal{K}$ on the affine subset $\left\{w_{2} \neq 0\right\}$ of $\mathbb{P}^{1}$.

We point out that the above form determines a class in the cohomology group $H^{2}\left(\mathbb{P}^{1}, \mathbb{Z}\right) \sim \mathbb{Z}$, which is the generator of $H^{2}\left(\mathbb{P}^{1}, \mathbb{Z}\right)$. Actually, the curvature form above is induced from the Fubini - Study metric on $\mathbb{P}^{1}$.

Let $\mathcal{M}_{0}$ be the largest submodule of functions in $\mathcal{M}$ vanishing at a fixed point $w \in \Omega \subseteq \mathbb{C}^{m}$. It is clear, in view of equation (2.6), that we will be able to construct a line bundle on the projective space $\mathbb{P}^{m-1}$ obtained by "blowing up" the point $w$.

To illustrate the ideas involved in the simplest possible terms, we assume that our domain is the bi-disc $\mathbb{D}^{2}$ and the submodule $\mathcal{M}_{0}$ is the closure of the ideal generated by the coordinate functions $z_{1}$ and $z_{2}$. Let $\theta_{1}$ and $\theta_{2}$ denote the usual homogeneous coordinates on the open sets $U$ and $V$ in the complex projective space $\mathbb{P}^{1}$ corresponding to the affine sets determined by $w_{1} \neq 0$ and $w_{2} \neq 0$ in $\mathbb{C}^{2}$. Let $E$ be the holomorphic line bundle on $\mathbb{P}^{1}$ defined, in local coordinates, by the following sections

$$
\begin{aligned}
& s_{1}\left(\theta_{1}\right)=\left.\left(\bar{\partial}_{1} K_{0}(\cdot, w)\right)\right|_{w=0}+\left.\theta_{1}\left(\bar{\partial}_{2} K_{0}(\cdot, w)\right)\right|_{w=0} \\
& s_{2}\left(\theta_{2}\right)=\left.\theta_{2}\left(\bar{\partial}_{1} K_{0}(\cdot, w)\right)\right|_{w=0}+\left.\left(\bar{\partial}_{2} K_{0}(\cdot, w)\right)\right|_{w=0} .
\end{aligned}
$$

THEOREM 5.1. Let $\mathcal{M}_{0}$ and $\mathcal{M}_{0}^{\prime}$ be two modules consisting of holomorphic functions on $\mathbb{D}^{2}$ which are generated by the two coordinate functions, that is, both modules consist of functions vanishing at the origin. Assume that the localisations $\mathcal{M}_{0}(w)$ and $\mathcal{M}_{0}^{\prime}(w)$ are one dimensional away from the zero set. If the modules $\mathcal{M}_{0}$ and $\mathcal{M}_{0}^{\prime}$ are isomorphic (equivalent via a unitary module map), then the corresponding bundles $E$ and $E^{\prime}$ they determine on the projective space are equivalent.

Proof: Let $L: \mathcal{M}_{0}^{\prime} \rightarrow \mathcal{M}_{0}$ be a unitary module map. By our assumption, the localisations of $\mathcal{M}_{0}$ and $\mathcal{M}_{0}^{\prime}$ on $\mathbb{D}^{2} \backslash\{0\}$ are one dimensional and are spanned by the corresponding reproducing kernels. Recall that $M_{f}^{*} K_{0}^{\prime}(\cdot, w)=\overline{f(w)} K_{0}^{\prime}(\cdot, w)$ for $f \in \mathcal{A}\left(\mathbb{D}^{2}\right)$ and $w \neq 0$. Since $L$ intertwines the module actions, it follows that 


$$
\begin{aligned}
& M_{f}^{*}\left(L K_{0}^{\prime}(\cdot, w)\right)=\overline{f(w)} L K_{0}^{\prime}(\cdot, w) . \text { Hence } \\
& \qquad L K_{0}^{\prime}(\cdot, w)=\overline{g(w)} K_{0}(\cdot, w), \text { for } w \neq 0 .
\end{aligned}
$$

It then follows that $g$ must be holomorphic on $\mathbb{D}^{2} \backslash\{0\}$. Since $L$ is unitary, we also have

Therefore,

$$
\left\|K_{0}^{\prime}(\cdot, w)\right\|^{2}=|g(w)|^{2}\left\|K_{0}(\cdot, w)\right\|^{2}, w \neq 0
$$

$$
\partial \bar{\partial} \log \frac{K_{0}^{\prime}(w, w)}{K_{0}(w, w)}=\partial \bar{\partial} \log |g(w)|^{2}=0, w \neq 0 .
$$

Consider the following change of coordinates on $\mathbb{D}^{2} \backslash\{0\}$ :

$$
\left(w_{1}, w_{2}\right) \mapsto\left(\varrho, \theta_{1}\right), w_{1}=\varrho, w_{2}=\varrho \theta_{1} .
$$

We then find that $\partial_{\theta_{1}} \bar{\partial}_{\theta_{1}}=\left|w_{1}\right|^{2} \partial_{2} \bar{\partial}_{2}$. Consequently, it follows that

$$
\partial_{\theta_{1}} \bar{\partial}_{\theta_{1}} \log \left|g\left(\varrho, \theta_{1}\right)\right|^{2}=0 \text { for } \varrho \neq 0 \text {. }
$$

From the form of these kernel functions given in equation (2.6), it follows that $\lim _{\varrho \rightarrow 0}\left|g\left(\varrho, \theta_{1}\right)\right|^{2}$ exists and is a real analytic function of the real and imaginary parts of $\theta_{1}$. From equation (5.6), it follows that $\lim _{\varrho \rightarrow 0} g\left(\varrho, \theta_{1}\right)$ is holomorphic in $\theta_{1}$. If $s_{1}$ and $s_{1}^{\prime}$ are sections of $E$ and $E^{\prime}$ on $U \subseteq \mathbb{P}^{1}$, then we see that

$$
L s_{1}^{\prime}\left(\theta_{1}\right)=g\left(0, \theta_{1}\right) s_{1}\left(\theta_{1}\right),
$$

where $g\left(0, \theta_{1}\right)=\lim _{\varrho \rightarrow 0} g\left(\varrho, \theta_{1}\right)$. This is a consequence of the following observations. From Equation (5.5) and the factorisation of $K_{0}$ and $K_{0}^{\prime}$ described earlier, it follows that $|g(w)|$ has a finite limit at the origin. By the Riemann removable singularity theorem, it follows that $g$ extends to a holomorphic function on all of $\mathbb{D}^{2}$. By performing the necessary differentiations on Equation (5.4), noting that differentiation with respect to $w$ commutes with $L$ and using the boundedness of $g$, we obtain (5.7).

From the unitarity of $L$ it follows that

$$
\left\|s_{1}^{\prime}\left(\theta_{1}\right)\right\|^{2}=\left|g\left(0, \theta_{1}\right)\right|^{2}\left\|s_{1}\left(\theta_{1}\right)\right\|^{2}
$$

and consequently the bundles determined by $\mathcal{M}_{0}$ and $\mathcal{M}_{0}^{\prime}$ on $\mathbb{P}^{1}$ are equivalent.

We remark that it is possible to prove a theorem analogous to the one above without making the restrictive assumption that the generators be irreducible polynomials. Indeed, in the second family of examples below, the submodules are generated by the functions $z_{1}^{2}, z_{2}^{2}$ and $z_{1} z_{2}$.

We illustrate by means of some examples the nature of the invariant we obtain from the bundle $E$ that lives on the projective space.

As we have shown, the curvature of the bundle which lives on the projective space is an invariant for the submodule. While this curvature does provide an invariant, the family discussed in Example 5.2 consisting of holomorphic functions vanishing at 0 , shows that it is not a complete invariant. However, surprisingly enough, if we consider a similar family of examples (see Example 5.3), this time consisting of functions vanishing to a higher order at 0 , then it turns out that 
within this family it is a complete invariant. We intend to investigate the nature of this invariant, arising out of a "blow up" construction, further at a future date.

EXAMPLE 5.2. Let $\mathcal{H}_{1}$ be the functional Hilbert space on the disc with the reproducing kernel

$$
K_{1}\left(z_{1}, w_{1}\right)=\frac{1}{\left(1-z_{1} \bar{w}_{1}\right)^{\lambda}} .
$$

Similarly, let $\mathcal{H}_{2}$ be the functional Hilbert space on the disc with the reproducing kernel

$$
K_{2}\left(z_{2}, w_{2}\right)=\frac{1}{\left(1-z_{2} \bar{w}_{2}\right)^{\mu}} .
$$

Let $\mathcal{H}$ be the tensor product of $\mathcal{H}_{1}$ and $\mathcal{H}_{2}$. Then $\mathcal{H}$ is a functional Hilbert space on the bi-disc $\mathbb{D}^{2}$. The reproducing kernel $K$ of $\mathcal{H}$ is the product of $K_{1}$ and $K_{2}$. That is,

$$
K\left(z_{1}, z_{2} ; w_{1}, w_{2}\right)=\frac{1}{\left(1-z_{1} \bar{w}_{1}\right)^{\lambda}\left(1-z_{2} \bar{w}_{2}\right)^{\mu}} .
$$

Define $\mathcal{M}_{(p, q)}^{\lambda, \mu}$ to be the subspace of functions in $\mathcal{H}$ which vanish at the point $(p, q)$ in the bi-disc. Our goal is to decide when two modules in the set

$$
\left\{\mathcal{M}_{(p, q)}^{\lambda, \mu}:(p, q) \in \mathbb{D}^{2}, \lambda, \mu>0\right\}
$$

are isomorphic. We begin by observing that

$$
\operatorname{dim} \mathcal{M}_{(p, q)}^{\lambda, \mu}(w)= \begin{cases}1 & \text { for }\left(w_{1}, w_{2}\right) \neq(p, q) \\ 2 & \text { for }\left(w_{1}, w_{2}\right)=(p, q) .\end{cases}
$$

This implies that $\mathcal{M}_{(p, q)}^{\lambda, \mu}$ is not equivalent to $\mathcal{M}_{\left(p^{\prime}, q^{\prime}\right)}^{\lambda^{\prime}, \mu^{\prime}}$ if $(p, q) \neq\left(p^{\prime}, q^{\prime}\right)$. Therefore, we are reduced to considering submodules $\mathcal{M}_{(p, q)}^{\lambda, \mu}$ with fixed $(p, q) \in \mathbb{D}^{2}$.

To explicitly compute the reproducing kernel for $\mathcal{M}_{(p, q)}^{\lambda, \mu}$, we recall that for $\phi, \psi$ in the Möbius group, the operators

$$
\begin{aligned}
& \left(U_{\phi} f\right)(z)=\left(\phi^{\prime}(z)\right)^{\lambda / 2} f(\phi(z)) \text { for } f \in \mathcal{H}_{1} \\
& \left(V_{\psi} f\right)(z)=\left(\psi^{\prime}(z)\right)^{\mu / 2} f(\psi(z)) \text { for } f \in \mathcal{H}_{2}
\end{aligned}
$$

define projective unitary representations of the Möbius group on $\mathcal{H}_{1}$ and $\mathcal{H}_{2}$, respectively [22]. Choose $\phi$ (respectively, $\psi$ ) to be the element of the Möbius group which maps the origin to the point $p$ (respectively, $q$ ) in the disc. Let $S_{\phi, \psi}$ denote the unitary operator $U_{\phi} \otimes V_{\psi}$. The operator $S_{\phi, \psi}$ clearly maps the subspace of functions vanishing at 0 to those vanishing at $(p, q)$. Furthermore, if $\left\{v_{n}\right\}$ is an orthonormal basis for the subspace of functions in $\mathcal{H}$ which vanish at the origin, $\left\{S_{\phi, \psi} v_{n}\right\}$ is an orthonormal basis for the subspace of functions in $\mathcal{H}$ which vanish at the point $(p, q)$. There is a natural basis for the subspace of functions which vanish at the origin which is constructed from the complete orthogonal set $\left\{z_{1}^{\ell} z_{2}^{k}:(\ell, k) \neq(0,0)\right\}$. This, in turn, gives 
us an orthonormal basis for the subspace of functions which vanish at the point $(p, q)$, from which it is not hard to compute the reproducing kernel $K_{(p, q)}$ for $\mathcal{M}_{(p, q)}^{\lambda, \mu}$ :

$$
\begin{aligned}
K_{(p, q)}(z, w)=\left(\phi^{\prime}\left(z_{1}\right) \overline{\phi^{\prime}\left(w_{1}\right)}\right)^{\lambda / 2}\left(\psi^{\prime}\left(z_{2}\right) \overline{\psi^{\prime}\left(w_{2}\right)}\right)^{\mu / 2} & \\
& \times\left(K\left(\left(\phi\left(z_{1}\right), \psi\left(z_{2}\right)\right),\left(\phi\left(w_{1}\right), \psi\left(w_{2}\right)\right)-1\right),\right.
\end{aligned}
$$

where $z=\left(z_{1}, z_{2}\right), w=\left(w_{1}, w_{2}\right)$ are in $\mathbb{D}^{2}$.

We now have a situation that is analogous to the previous example. The procedure outlined there can be applied to "blow up" the point $(p, q)$ and to construct a line bundle on the projective space at $(p, q)$. We can calculate the section of this line bundle using the explicit form of the reproducing kernel $K_{(p, q)}$. The section $s_{1}$ on the coordinate patch $U$, as an element of $\mathcal{M}_{(p, q)}^{\lambda, \mu}$, is of the form

$$
\begin{aligned}
\left(s_{1}\left(\theta_{1}\right)\right)\left(z_{1}, z_{2}\right)= & \frac{\lambda}{1-|p|^{2}}\left(\frac{\phi\left(z_{1}\right)}{\left(1-\bar{p} z_{1}\right)^{\lambda}\left(1-\bar{q} z_{2}\right)^{\mu}}\right) \\
& +\theta_{1} \frac{\mu}{1-|q|^{2}}\left(\frac{\psi\left(z_{2}\right)}{\left(1-\bar{p} z_{1}\right)^{\lambda}\left(1-\bar{q} z_{2}\right)^{\mu}}\right) .
\end{aligned}
$$

The curvature of this line bundle turns out to be the $(1,1)$-form with coefficient

$$
C_{\lambda, \mu}\left(\theta_{1}\right)=\frac{\left(1-|q|^{2}\right)^{2}\left(1-|p|^{2}\right)^{2} \mu \lambda}{\left\{\left(1-|q|^{2}\right)^{2} \lambda+\left(1-|p|^{2}\right)^{2} \mu\left|\theta_{1}\right|^{2}\right\}^{2}} .
$$

In case $(p, q)=(0,0)$, we have $C_{\lambda, \mu}\left(\theta_{1}\right)=\frac{\lambda \mu}{\left(\lambda+\mu\left|\theta_{1}\right|^{2}\right)^{2}}$. We get that $C_{\lambda, \mu}=$ $C_{\lambda^{\prime}, \mu^{\prime}}$ if $\frac{\lambda}{\mu}=\frac{\lambda^{\prime}}{\mu^{\prime}}$ even though $(\lambda, \mu) \neq\left(\lambda^{\prime}, \mu^{\prime}\right)$. (Of course, from the general formula for the curvature given above, it is easy to see that we get a similar result at an arbitrary point.) In all probability the submodules corresponding to two distinct pairs $(\lambda, \mu)$ and $\left(\lambda^{\prime}, \mu^{\prime}\right)$ are not equivalent.

Theorem 5.1 was stated for the case of functions which vanish at the origin in $\mathbb{D}^{2}$ but can naturally be generalised to any other point. There is also a generalisation of this theorem to functions which vanish to higher order at a point. We present a brief outline for the case of functions which vanish to order 2 and illustrate the usefulness of the result by means of an example.

The sections $s_{1}$ and $s_{2}$ are now given by

$$
\begin{aligned}
& s_{1}\left(\theta_{1}\right)=\left.\left(\left(\bar{\partial}_{1}+\theta_{1} \bar{\partial}_{2}\right)^{2} K_{0}(\cdot, w)\right)\right|_{w=0} \\
& s_{2}\left(\theta_{2}\right)=\left.\left(\left(\theta_{2} \bar{\partial}_{1}+\bar{\partial}_{2}\right)^{2} K_{0}(\cdot, w)\right)\right|_{w=0} .
\end{aligned}
$$

A result analogous to Theorem 5.1 holds for the line bundle that these sections determine on the projective space $\mathbb{P}^{1}$. As mentioned earlier, the curvature of this line bundle gives a complete invariant for the family of modules considered in the example below.

EXAMPLE 5.3. The setting of this example is the same as that of Example 5.2 and makes use of the Hilbert spaces $\mathcal{H}_{1}, \mathcal{H}_{2}$ and $\mathcal{H}$ defined there. 
We let $\mathcal{N}_{(p, q)}^{\lambda, \mu}$ be the subspace of functions in $\mathcal{H}$ which vanish to order 2 at the point $(p, q)$ in the bi-disc. The dimension of the localisation of $\mathcal{N}_{(p, q)}^{\lambda, \mu}$ jumps at the point $(p, q)$ and hence $\mathcal{N}_{(p, q)}^{\lambda, \mu}$ is not equivalent to $\mathcal{N}_{\left(p^{\prime}, q^{\prime}\right)}^{\lambda^{\prime}, \mu^{\prime}}$ if $(p, q) \neq\left(p^{\prime}, q^{\prime}\right)$

As in Example 5.2 we now consider the family of modules $\mathcal{N}_{(0,0)}^{\lambda, \mu}$. The section $s_{1}$ of the line bundle on $\mathbb{P}^{1}$ is given (as an element of $\mathcal{N}_{(0,0)}^{\lambda, \mu}$ ) by

$$
\left(s_{1}\left(\theta_{1}\right)\right)(z)=\lambda(\lambda+1) z_{1}^{2}+2 \lambda \mu \theta_{1} z_{1} z_{2}+\mu(\mu+1) \theta_{1}^{2} z_{2}^{2}
$$

The squared norm of the above section is

$$
2 \lambda(\lambda+1)+4 \lambda \mu\left|\theta_{1}\right|^{2}+2 \mu(\mu+1)\left|\theta_{1}\right|^{4}
$$

and consequently the curvature is

where

$$
\mathcal{K}\left(\theta_{1}\right)=\frac{b+4 c\left|\theta_{1}\right|^{2}+b c\left|\theta_{1}\right|^{4}}{\left(1+b\left|\theta_{1}\right|^{2}+c\left|\theta_{1}\right|^{4}\right)^{2}}
$$

$$
b=\frac{\mu(\mu+1)}{\lambda(\lambda+1)} \text { and } c=\frac{\mu}{\lambda+1} .
$$

It follows that two modules in this family corresponding to parameter values $(\lambda, \mu)$ and $\left(\lambda^{\prime}, \mu^{\prime}\right)$ are equivalent if and only if the corresponding values of $b$ and $c$ are equal. A simple computation shows that this happens if and only if $(\lambda, \mu)=\left(\lambda^{\prime}, \mu^{\prime}\right)$, showing that the curvature of the line bundle on the projective space is a complete invariant for modules in this family.

We emphasise the advantage of using the bundle that we have constructed on the projective space obtained by blowing up the zero set (the origin in our examples). It is conceivable that the inequivalence results obtained above can also be obtained by localising on $\mathbb{D}^{2} \backslash\{0\}$ and looking at the curvature of the corresponding line bundle as in the Cowen-Douglas theory. However, any attempt to do so, even in the simple Examples 5.2, lead to complicated calculations. Part of the complication is due to the fact that this latter bundle lives on a two dimensional base space. As illustrated above, in the Examples 5.3, it often suffices to consider only the bundle defined on the projective space.

Many of the issues that we have raised in the context of Hilbert modules have been studied in analogous algebraic settings. We have attempted to adapt some of these results and techniques. Resolutions of modules have been studied intensely during the last half of the twentieth century in both the algebraic and analytic settings. This has been extended to the context of complex geometry - in the presence of a hermitian metric - only in the last couple of decades. The work of Bismut-Gillet-Soulé and Harvey-Lawson, cited earlier, are examples of this effort, but not the only ones. The whole of what is called Arakelov theory falls in this area. The problems which are described above are, we believe, both interesting and important.

Acknowledgement: The authors would like to thank B. Bagchi and V. Pati for many hours of helpful conversations. 


\section{References}

[1] N. Aronszajn, Theory of reproducing kernels, Trans. Amer.Math. Soc., 68 (1950), $337-404$.

[2] J. M. Bismut, H. Gillet and C. Soulé, Analytic torsion and holomorphic determinant bundles, I, II and III, Comm. Math. Phy., 115 (1988), 49 - 78, 79 - 126, 301 - 351.

[3] R. Bott and S. S. Chern, Hermitian vector bundles and the equidistribution of the zeros of their holomorphic sections, Acta Math., 114 (1968), 71 - 112.

[4] X. Chen and R. G. Douglas, Localization of Hilbert Modules, Michigan Math. J., 39 (1992), 443 - 454.

[5] D. N. Clark, Restrictions of $H^{p}$ functions in the polydisk, Amer. J. Math., 110 (1988), 1119 - 1152.

[6] M. Cowen and R. G. Douglas, Complex geometry and operator theory, Acta Math., 141 (1978), 187-261.

[7] M. Cowen and R. G. Douglas, On operators possessing an open set of eigenvalues, Memorial conf. for Féjer-Riesz, Budapest, Colloq. Math. Soc. J. Bolyai, (1980), 323 $-341$.

[8] Jean-Pierre Demailly and Mikael Passare, Courants résiduels et classe fondamentale, Bull. Sci. Math., 119 (1995), 85 - 94.

[9] Jean-Pierre Demailly, Multiplier ideal sheaves and analytic methods in algebraic geometry, Lecture Notes, School on vanishing theorems and effective results in algebraic geometry, ICTP, Trieste, April 2000.

[10] R. E. Curto and N. Salinas, Generalized Bergman kernels and the Cowen-Douglas theory, Amer J. Math., 106(1984), 447 - 488.

[11] R. G. Douglas and G. Misra, Some Calculations for Hilbert Modules, J. Orissa Math. Soc., 12-15 (1993-96), 75 - 85.

[12] R. G. Douglas and G. Misra, Geometric invariants for resolutions of Hilbert modules, Operator Theory: Advances and Applications, 104 (1998), 83 - 112.

[13] Ronald G. Douglas and Gadadhar Misra, On quotient modules, Operator Theory: Advances and Applications, special volume dedicated to the memory of Bela SzokefalviNagy, to appear.

[14] Ronald G. Douglas, Gadadhar Misra and Cherian Varughese, On quotient modules - The case of arbitrary multiplicity, J. Functional Anal., 174 (2000), 364 - 398.

[15] R. G. Douglas and V. I. Paulsen, Hilbert Modules over Function Algebras, Longman Research Notes, 217, 1989.

[16] Donggeng Gong and Joel Pincus, Torsion invariants for finite von Neumann algebras, Contemporary Mathematics, 185 (1995), 151 - 186.

[17] Phillip Griffiths and James King, Nevanlinna theory and holomorphic mappings between algebraic varieties, Acta Math., 130 (1973), 145 - 220.

[18] R. C. Gunning and H. Rossi, Analytic functions of several complex variables, Prentice Hall, New York, 1965.

[19] F. R. Harvey and H. B. Lawson, A theory of characteristic currents associated with a singular connection, Asterisque, 213 (1993), 1 - 268.

[20] M. Martin and N. Salinas, Flag Manifolds and the Cowen-Douglas Theory, J. Operator Theory, 38 (1997), 329 - 365. 
[21] B. Sz-Nagy and C. Foias, Harmonic Analysis of operators on Hilbert space, North Holland, 1970.

[22] P. J. Sally, Analytic continuation of the irreducible unitary representations of the universal covering group of $S L(2, \mathbb{R})$, Mem. Amer. Math. Soc. 69, Providence, 1967.

[23] R. O. Wells, Jr., Differential analysis on complex manifolds, Springer Verlag, 1973.

Texas A\&M University, College Station, Texas 77843

E-mail address, Ronald G. Douglas: rgd@tamu.edu

Indian Statistical Institute, R. V. College Post, Bangalore 560059

E-mail address, Gadadhar Misra: gm@isibang.ac.in

E-mail address, Cherian Varughese: cherian@isibang.ac.in 